\title{
Review \\ Balneotherapy for Fibromyalgia Syndrome: A Systematic Review and Meta-Analysis
}

\author{
Chun-Feng Cao ${ }^{1,+}{ }^{\dagger}$ Kun-Long Ma ${ }^{1,+}{ }^{\dagger}$,ian-Lu Li ${ }^{2}$, Fu-Jun Luan ${ }^{1}$, Qun-Bo Wang ${ }^{1}$, Ming-Hua Zhang ${ }^{1}$, \\ Omar Viswanath ${ }^{3,4,5,6}$, Dariusz Myrcik ${ }^{7}$, Giustino Varrassi ${ }^{8, *}$ al and Hai-Qiang Wang $9, *$ (i)
}

1 Department of Orthopedics, The Yongchuan Hospital of Chongqing Medical University, 439\# Xuanhua Road, Yongchuan, Chongqing 402160, China; 711204@hospital.cqmu.edu.cn (C.-F.C.); 701079@hospital.cqmu.edu.cn (K.-L.M.); 701148@hospital.cqmu.edu.cn (F.-J.L.); 700009@hospital.cqmu.edu.cn (Q.-B.W.); 700280@hospital.cqmu.edu.cn (M.-H.Z.)

2 Department of Neurology, The Yongchuan Hospital of Chongqing Medical University, 439\# Xuanhua Road, Yongchuan, Chongqing 402160, China; 710197@hospital.cqmu.edu.cn

3 Department of Anesthesiology, Creighton University School of Medicine, Omaha, NE 68114, USA; viswanoy@gmail.com

4 Department of Anesthesiology, University of Arizona College of Medicine-Phoenix, Phoenix, AZ 85003, USA

5 Department of Anesthesiology, Louisiana State University Shreveport, Shreveport, LA 71106, USA

6 Department of Pain Management, Valley Pain Consultants-Envision Physician Services, Phoenix, AZ 85003, USA

7 Department of Internal Medicine, Medical University of Silesia, Katowice, 42-600 Bytom, Poland; dariuszmyrcik@me.com

check for updates

Citation: Cao, C.-F.; Ma, K.-L.; Li, Q.-L.; Luan, F.-J.; Wang, Q.-B.; Zhang, M.-H.; Viswanath, O.; Myrcik, D.; Varrassi, G.; Wang, H.-Q. Balneotherapy for Fibromyalgia Syndrome: A Systematic Review and Meta-Analysis. J. Clin. Med. 2021, 10, 1493. https://doi.org/10.3390/ jcm10071493

Academic Editors: Won Ho Kim and Katarzyna Kotfis

Received: 22 January 2021

Accepted: 31 March 2021

Published: 3 April 2021

Publisher's Note: MDPI stays neutral with regard to jurisdictional claims in published maps and institutional affiliations.

Copyright: (c) 2021 by the authors. Licensee MDPI, Basel, Switzerland. This article is an open access article distributed under the terms and conditions of the Creative Commons Attribution (CC BY) license (https:/ / creativecommons.org/licenses/by/ $4.0 /)$.
8 Department of Research, Polo Procacci Foundation, via Tacito 7, 00193 Roma, Italy

9 Institute of Integrative Medicine, Shaanxi University of Chinese Medicine, Xixian Avenue, Xixian District, Xi'an 712046, China

* Correspondence: giuvarr@gmail.com (G.V.); hqwang@sntcm.edu.cn (H.-Q.W.)

+ These authors contributed equally and should be considered as co-first authors.

Abstract: (1) Background: The efficiency of balneotherapy (BT) for fibromyalgia syndrome (FMS) remains elusive. (2) Methods: Cochrane Library, EMBASE, MEDLINE, PubMed, Clinicaltrials.gov, and PsycINFO were searched from inception to 31 May 2020. Randomized controlled trials (RCTs) with at least one indicator were included, i.e., pain, Fibromyalgia Impact Questionnaire (FIQ), Tender Points Count (TPC), and Beck's Depression Index (BDI). The outcome was reported as a standardized mean difference (SMD), 95\% confidence intervals (CIs), and $I^{2}$ for heterogeneity at three observational time points. GRADE was used to evaluate the strength of evidence. (3) Results: Amongst 884 citations, 11 RCTs were included $(n=672)$. Various BT regimens were reported (water types, duration, temperature, and ingredients). BT can benefit FMS with statistically significant improvement at different time points (pain of two weeks, three and six months: SMD $=-0.92,-0.45$, $-0.70 ; 95 \%$ CI $(-1.31$ to $-0.53,-0.73$ to $-0.16,-1.34$ to -0.05$) ; I^{2}=54 \%, 51 \%, 87 \%$; GRADE: very low, moderate, low; FIQ: SMD $=-1.04,-0.64,-0.94 ; 95 \%$ CI $(-1.51$ to $-0.57,-0.95$ to $-0.33,-1.55$ to -0.34$) ; I^{2}=76 \%, 62 \%, 85 \%$; GRADE: low, low, very low; TPC at two weeks and three months: SMD $=-0.94,-0.47 ; 95 \%$ CI $(-1.69$ to $-0.18,-0.71$ to -0.22$) ; I^{2}=81 \%, 0$; GRADE: very low, moderate; BDI at six months: $\mathrm{SMD}=-0.45 ; 95 \% \mathrm{CI}(-0.73$ to -0.17$) ; I^{2}=0$; GRADE: moderate). There was no statistically significant effect for the TPC and BDI at the remaining time points (TPC at six months: SMD $=-0.89 ; 95 \%$ CI $(-1.85$ to 0.07$) ; I^{2}=91 \%$; GRADE: very low; BDI at two weeks and three months: $\mathrm{SMD}=-0.35,-0.23 ; 95 \% \mathrm{CI}(-0.73$ to $0.04,-0.64$ to 0.17$) ; I^{2}=24 \%, 60 \%$; GRADE: moderate, low). (4) Conclusions: Very low to moderate evidence indicates that BT can benefit FMS in pain and quality-of-life improvement, whereas tenderness and depression improvement varies at time phases. Established BT regimens with a large sample size and longer observation are needed.

Keywords: balneotherapy; fibromyalgia; meta-analysis; systematic review; musculoskeletal disease 


\section{Introduction}

Fibromyalgia syndrome (FMS) is a musculoskeletal disorder characterized by widespread skeletal muscle pain. It is also considered a chronic pain syndrome mainly due to the dysfunction of the central nervous system [1]. Patients can present with various accompanied symptoms, including fatigue, sleep disturbances, headache, morning stiffness, anxiety, and depression [2,3]. The prevalence of FMS is estimated at $1-2 \%$ in the general population. The disorder mainly affects women, with an incidence rate six times higher than that of men [4]. Its treatment is usually complex [5] and unsatisfactory, thus being identified as a public health burden due to significant health expenses for the treatment of the musculoskeletal disease, including FMS [6]. Consequently, FMS patients are responsible for a high rate of medical consultations and a high consumption of drugs [7].

Balneotherapy (BT) is a non-invasive alternative treatment to relieve musculoskeletal or neuropathic pain and stiffness, improving the quality of life amongst the elderly with musculoskeletal pain [8]. Despite BT being defined as bathing in natural mineral waters, spas, or cures as a Medical subject Headings (MeSH) term (MeSH Unique ID: D001452), the practice mode varies greatly in the literature, including the temperature of the waters, therapy duration/sessions, mineral contents, and concentrations [9-11]. Previous randomized clinical trials (RCTs), meta-analyses, and systematic reviews have shown its effectiveness in alleviating symptoms of patients with musculoskeletal disorders [12,13]. However, studies on BT for FMS are disputable with a potential source of bias, such as a lack of double-blinded studies $[14,15]$. So far, there has been a paucity of randomized evidence investigating the efficiency of BT for FMS. A previous study has evaluated the issue, with limited outcome indicators [9]. Moreover, new studies on BT for FMS have not been successfully analyzed by systematic reviews until now. Therefore, we conducted an updated systematic review and meta-analysis to determine whether the existing data show the efficacy of BT in the treatment of FMS.

\section{Methods}

Given that the study was a systematic review, ethics committee approval was waived.

\subsection{Protocol and Registration}

This systematic review and meta-analysis was performed according to the Preferred Reporting Items for Systematic Review and Meta-Analysis Protocols 2015 (PRISMA-P) guidelines [16]. It has been registered in the International Prospective Register of Systematic Reviews (PROSPERO), with the registration number CRD42019142187.

\subsection{Search Strategy}

We searched the Cochrane Library, EMBASE, MEDLINE, PubMed, Clinicaltrials.gov, and PsycINFO thoroughly from their inception to 31 May 2020 to quantitatively compare the pooled effect of BT for FMS. The searching strategies were worked out by two reviewers (C.F.C. and K.L.M.) who were experienced to identify relevant studies. We adopted the search strategies "(FMS OR fibromyalgia OR musculoskeletal disease) AND (BT OR spa therapy OR balneotherapy OR balneology OR thermal water)" without language restrictions (Table 1). The search filter was limited to randomized clinical trials (RCTs). In addition, the reference lists of relevant articles were reviewed for further papers to be included. 
Table 1. Search strategy and results.

\begin{tabular}{|c|c|c|c|}
\hline Database & Step & Search Algorithm & Items Found \\
\hline \multirow{12}{*}{ PubMed } & $\# 1$ & "fibromyalgia" [Mesh] & 8416 \\
\hline & $\# 2$ & "musculoskeletal disease" & 1718 \\
\hline & $\# 3$ & "chronic pain syndrome" & 675 \\
\hline & $\# 4$ & “FMS" & 9012 \\
\hline & $\# 5$ & \#1 OR \#2 OR \#3 OR \#4 & 21,981 \\
\hline & \#6 & "balneotherapy" & 1397 \\
\hline & $\# 7$ & "spa therapy" & 314 \\
\hline & $\# 8$ & "thermal water" & 298 \\
\hline & $\# 9$ & "balneology" & 6116 \\
\hline & $\# 10$ & "BT" & 23,370 \\
\hline & $\# 11$ & \#6) OR \#7 OR \#8 OR \#9 OR \#10 & 25,239 \\
\hline & $\# 12$ & \#5 AND \#11 & 99 \\
\hline \multirow{12}{*}{ Embase } & $\# 1$ & 'fibromyalgia' /exp & 20,842 \\
\hline & $\# 2$ & 'musculoskeletal disease' & 35,937 \\
\hline & $\# 3$ & 'chronic pain syndrome' & 1133 \\
\hline & $\# 4$ & ‘FMS' & 12,213 \\
\hline & $\# 5$ & \#1 OR \#2 OR \#3 OR \#4 & 67,541 \\
\hline & \#6 & "balneotherapy" /exp & 16,620 \\
\hline & $\# 7$ & "spa therapy" & 602 \\
\hline & $\# 8$ & "thermal water" & 484 \\
\hline & $\# 9$ & "balneology" & 1471 \\
\hline & $\# 10$ & “BT" & 35,106 \\
\hline & $\# 11$ & \#6 OR \#7 OR \#8 OR \#9 OR \#10 & 52,736 \\
\hline & $\# 12$ & \#5 AND \#11 & 434 \\
\hline \multirow{12}{*}{ Cochrane } & $\# 1$ & MeSH descriptor: [fibromyalgia] explode all trees & 2927 \\
\hline & $\# 2$ & $\begin{array}{l}\text { musculoskeletal disease: } \mathrm{ti}, \mathrm{ab}, \mathrm{kw} \text { (Word variations have been } \\
\text { searched) }\end{array}$ & 4114 \\
\hline & $\# 3$ & $\begin{array}{l}\text { chronic pain syndrome: ti,ab,kw (Word variations have been } \\
\text { searched) }\end{array}$ & 280 \\
\hline & $\# 4$ & FMS: ti,ab,kw (Word variations have been searched) & 649 \\
\hline & $\# 5$ & $\# 1$ or \#2 or \#3 or \#4 & 7576 \\
\hline & \#6 & MeSH descriptor: [balneotherapy] explode all trees & 261 \\
\hline & $\# 7$ & spa therapy: ti,ab,kw (Word variations have been searched) & 122 \\
\hline & $\# 8$ & thermal water: ti,ab,kw (Word variations have been searched) & 79 \\
\hline & $\# 9$ & nalneology: ti,ab,kw (Word variations have been searched) & 211 \\
\hline & $\# 10$ & BT: ti,ab,kw (Word variations have been searched) & 1373 \\
\hline & $\# 11$ & $\# 6$ or $\# 7$ or $\# 8$ or $\# 9$ or $\# 10$ & 1848 \\
\hline & $\# 12$ & $\# 5$ and \#11 restricted as clinical trials & 52 \\
\hline
\end{tabular}


Table 1. Cont.

\begin{tabular}{|c|c|c|c|}
\hline Database & Step & Search Algorithm & Items Found \\
\hline \multirow{12}{*}{ Medline } & $\# 1$ & "fibromyalgia" & 6926 \\
\hline & $\# 2$ & "musculoskeletal disease" & 6294 \\
\hline & $\# 3$ & "chronic pain syndrome" & 329 \\
\hline & $\# 4$ & “FMS" & 7109 \\
\hline & $\# 5$ & $\begin{array}{l}\text { fibromyalgia OR musculoskeletal disease OR chronic pain } \\
\text { syndrome OR FMS }\end{array}$ & 26,976 \\
\hline & $\# 6$ & "balneotherapy" & 472 \\
\hline & $\# 7$ & "spa therapy" & 273 \\
\hline & $\# 8$ & "thermal water" & 2131 \\
\hline & $\# 9$ & "nalneology" & 562 \\
\hline & $\# 10$ & "BT" & 21,713 \\
\hline & $\# 11$ & $\begin{array}{l}\text { nalneotherapy OR spa therapy OR thermal water OR balneology } \\
\text { OR BT }\end{array}$ & 38,850 \\
\hline & $\# 12$ & $\# 5$ and \#11 & 91 \\
\hline \multirow{12}{*}{ PsycINFO } & $\# 1$ & "fibromyalgia" & 46 \\
\hline & $\# 2$ & "musculoskeletal disease" & 26 \\
\hline & $\# 3$ & "chronic pain syndrome" & 47 \\
\hline & $\# 4$ & “FMS" & 19 \\
\hline & $\# 5$ & $\begin{array}{l}\text { fibromyalgia OR musculoskeletal disease OR chronic pain } \\
\text { syndrome OR FMS }\end{array}$ & 116 \\
\hline & \#6 & "nalneotherapy" & 0 \\
\hline & $\# 7$ & "spa therapy" & 1 \\
\hline & $\# 8$ & "thermal water" & 13 \\
\hline & $\# 9$ & "nalneology" & 1 \\
\hline & $\# 10$ & "BT" & 0 \\
\hline & $\# 11$ & $\begin{array}{l}\text { nalneotherapy OR spa therapy OR thermal water OR balneology } \\
\text { OR BT }\end{array}$ & 15 \\
\hline & $\# 12$ & $\# 5$ and \#11 & 1 \\
\hline \multirow{12}{*}{ Clinicaltrials.gov } & $\# 1$ & "fibromyalgia" & 958 \\
\hline & $\# 2$ & "musculoskeletal disease" & 17,031 \\
\hline & $\# 3$ & "chronic pain syndrome" & 44 \\
\hline & $\# 4$ & "FMS" & 1026 \\
\hline & $\# 5$ & $\begin{array}{c}\text { fibromyalgia OR musculoskeletal disease OR Chronic pain } \\
\text { syndrome OR FMS }\end{array}$ & 17,308 \\
\hline & $\# 6$ & "balneotherapy" & 16 \\
\hline & $\# 7$ & "spa therapy" & 22 \\
\hline & $\# 8$ & "thermal water" & 3 \\
\hline & $\# 9$ & "balneology" & 6 \\
\hline & $\# 10$ & “BT" & 45 \\
\hline & $\# 11$ & $\begin{array}{l}\text { balneotherapy OR spa therapy OR thermal water OR balneology } \\
\text { OR BT }\end{array}$ & 107 \\
\hline & $\# 12$ & $\begin{array}{c}\text { balneotherapy OR spa therapy OR thermal water OR balneology } \\
\text { OR BT I fibromyalgia OR musculoskeletal disease OR chronic } \\
\text { pain syndrome OR FMS }\end{array}$ & 207 \\
\hline
\end{tabular}




\subsection{Inclusion and Exclusion Criteria}

Studies were required to meet the following inclusion criteria: (1) RCTs with FMS diagnosis based on the American College of Rheumatology (ACR) criteria [17,18]; (2) type of intervention as comparing BT with no treatment or other treatment options; (3) outcome measures reporting at least one FMS symptom-related item, including pain, Tender Points Count (TPC), Beck's Depression Index (BDI), and Fibromyalgia Impact Questionnaire (FIQ); and (4) publication of the study in full paper form.

We excluded retrospective studies, cohort studies, and clinical controlled studies. Two authors (C.-F.C. and K.-L.M.) screened the studies independently. An initial screening of titles was performed, with duplicates excluded. Subsequently, full texts and abstracts were reviewed and irrelevant papers were removed.

\subsection{Data Extraction and Management}

Data were extracted from all the studies meeting the inclusion criteria, and following an instruction manual. For all articles, two authors (Q.-L.L. and F.-J.L.) reviewed data independently. Potential disagreements were solved by consensus. When consensus could not be reached, a third author (M.-H.Z.) was required to extract data and discuss for consensus. When data were reported as a median (low-high), the mean and variance were calculated using the appropriate formula [19].

\subsection{Assessment of Risk of Bias (ROB) in Included Studies}

Two authors (C.-F.C. and K.-L.M.) assessed the ROBs of each study independently according to the Cochrane Collaboration's ROB 2 tool [20]. ROB 2 includes optional judgments of the direction of bias for each domain and overall. We applied the form of ROB assessment, including five key indicators: randomization process, intended interventions, missing outcome data, the measurement of the outcome, and the selection of reported results. All disagreements were resolved by consensus and eventually the consultation of a third author (Q.-B.W.).

\subsection{Assessment of Treatment Effect}

Meta-analysis was performed using Review Manager 5.3 software. The standardized mean difference (SMD) was calculated using a random-effects model, when data were continuous, and $95 \%$ confidence intervals (CIs) were determined for all effect sizes. The strength of evidence was assessed using the online version of GRADEpro GDT software, identified as high, moderate, low, and very low [21].

\subsection{Assessment of Heterogeneity}

Heterogeneity between comparable trials was analyzed using standard Cochran's Q tests and the $I^{2}$ statistic before meta-analysis. $I^{2}$ values were taken according to Deeks [22] A $p$-value of $<0.05$ was considered statistically significant.

\subsection{Sensitivity Analysis}

Sensitivity analysis was performed for high-risk and low-risk bias studies, studies with serious deficiencies in one or more key areas, and the sample size for each treatment group.

\section{Results}

\subsection{Hallmarks of Included Studies}

The literature-retrieving strategy and pertaining results are shown in Figure 1. A total of 884 relevant studies were preliminarily reviewed (PubMed search, 99 citations; Cochrane search, 52 citations; EMBASE search, 434 citations; Medline search, 91 citations; PsycINFO search, 1 citation; and Clinicaltrail.gov search, 207 citations). In total, 13 RCT studies eventually satisfied the eligibility criteria, and 11 were included for this meta-analysis. After the initial screening, one study was excluded, given that the means and standard 
deviation of post-test data were not reported and could not be calculated [23]. Another study was excluded because the exercises in the pool were used as therapy, and not only the bath [24].

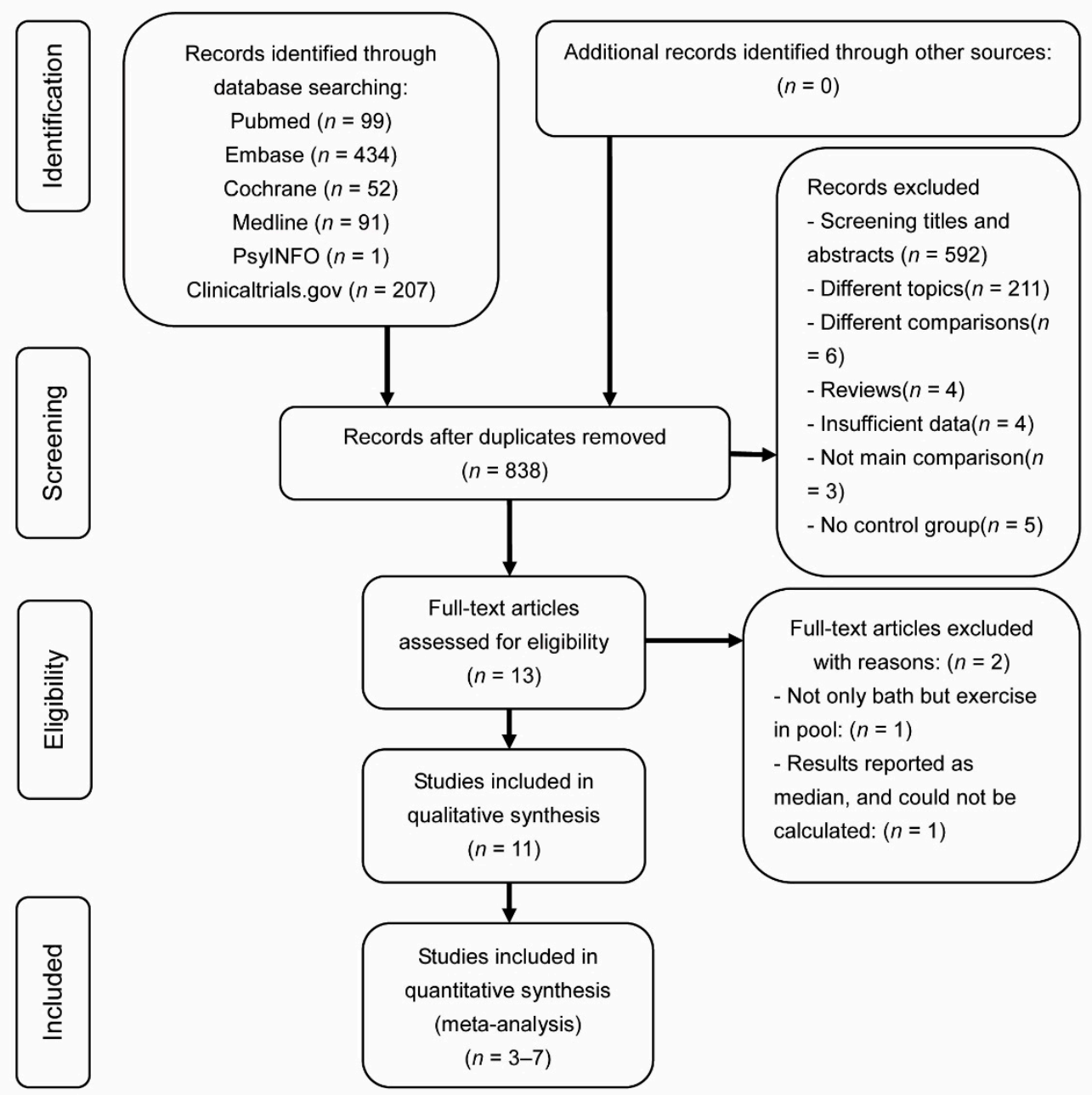

Figure 1. Flowchart of literature-screening process.

\subsection{Location Hallmarks}

Included RCTs were mainly derived from Europe and Asia. In detail, six studies were from Turkey [8,25-29], three from Italy [30-32], one from Spain [33], and one from the Netherlands [34].

\subsection{Participant Hallmarks}

In total, 672 participants were included, with 330 patients undergoing BT intervention and 342 being controls. The average age was 46.1 years in the BT group (range, 42.0 to 56.2 years) and 45.7 years in the control group (range, 41.5 to 55.9 years). The gender was reported in nine studies. Females were highly prevalent (287 females and 15 males in the BT group, 306 females and 14 males in the control group). The average observational time phase was 19.7 weeks (range, 3 to 48 weeks). The average disease duration was 5.13 years according to data in nine studies (range, 1.3 to 12.9 years). A detailed list of study hallmarks is shown in Table 2. 
Table 2. Detailed information and characteristics of included studies.

\begin{tabular}{|c|c|c|c|c|c|c|c|c|c|c|}
\hline \multirow{2}{*}{ Studies } & \multirow{2}{*}{ Country } & \multicolumn{2}{|c|}{ Age (y) } & \multicolumn{2}{|c|}{ Female/Male } & \multicolumn{2}{|c|}{ Sample Size $(n)$} & \multicolumn{2}{|c|}{ Duration } & \multirow{2}{*}{ Follow-Up (w) } \\
\hline & & BT & con & BT & con & BT & con & BT & con & \\
\hline $\begin{array}{c}\text { Fioravanti et al., } \\
2018 \text { [32] }\end{array}$ & Italy & $56.16 \pm 8.74$ & $55.9 \pm 6.61$ & $48 / 2$ & $47 / 3$ & 50 & 50 & $16.02 \pm 12.58 \mathrm{~m}$ & $15.08 \pm 10.87 \mathrm{~m}$ & 24 \\
\hline $\begin{array}{l}\text { Fernández et al., } \\
2019 \text { [33] }\end{array}$ & Spain & $52.4 \pm 8.6$ & $53.4 \pm 11.3$ & $23 / 2$ & $25 / 0$ & 25 & 25 & NA & NA & 12 \\
\hline $\begin{array}{c}\text { Dönmez et al., } \\
2005 \text { [26] }\end{array}$ & Turkey & $43.3 \pm 7.5$ & $43.1 \pm 6.9$ & NA & NA & 16 & 13 & $11.5 \pm 8.5 y$ & $11.8 \pm 7.5 \mathrm{y}$ & 36 \\
\hline $\begin{array}{l}\text { Özkurt et al., } \\
2012 \text { [30] }\end{array}$ & Italy & $50.8 \pm 6$ & $46.87 \pm 8.8$ & $21 / 0$ & $24 / 0$ & 21 & 24 & $12.9 \pm 7 y$ & $11.29 \pm 6.2 \mathrm{y}$ & 12 \\
\hline $\begin{array}{l}\text { Zijlstra et al., } \\
2005 \text { [34] }\end{array}$ & $\begin{array}{l}\text { The } \\
\text { Nether- } \\
\text { lands }\end{array}$ & 48 & 47 & $55 / 3$ & $73 / 3$ & 58 & 76 & $10 \mathrm{~m}$ & $10 \mathrm{~m}$ & 48 \\
\hline $\begin{array}{l}\text { Bağdatl et al., } \\
2015 \text { [29] }\end{array}$ & Turkey & $45.17 \pm 9.09$ & $42.77 \pm 9.59$ & $35 / 0$ & $35 / 0$ & 35 & 35 & $8.83 \pm 4.74 y$ & $8.37 \pm 5.49 y$ & 12 \\
\hline $\begin{array}{l}\text { Evcik et al., } \\
2002 \text { [25] }\end{array}$ & Turkey & $42 \pm 6.8$ & $41.5 \pm 7.1$ & $16 / 6$ & $15 / 5$ & 22 & 20 & $15.5 \pm 7.2 \mathrm{~m}$ & $14.1 \pm 8.7 \mathrm{~m}$ & 24 \\
\hline $\begin{array}{l}\text { Ardıç et al., } \\
2007 \text { [27] }\end{array}$ & Turkey & $43.5 \pm 10.2$ & $48.8 \pm 8.8$ & NA & NA & 12 & 9 & $23.4 \pm 21.5 \mathrm{~m}$ & $30.6 \pm 28.6 \mathrm{~m}$ & 3 \\
\hline $\begin{array}{c}\text { Yurtkuran et al., } \\
1996 \text { [8] }\end{array}$ & Turkey & 37.5 & 33.4 & $19 / 1$ & $18 / 2$ & 20 & 20 & NA & NA & 6 \\
\hline $\begin{array}{c}\text { Fioravanti et al., } \\
2007 \text { [31] }\end{array}$ & Italy & $46.2 \pm 10.5$ & $48.6 \pm 9.4$ & $39 / 1$ & $39 / 1$ & 40 & 40 & $2.21 \pm 1.35 \mathrm{y}$ & $2.30 \pm 1.42 \mathrm{y}$ & 16 \\
\hline $\begin{array}{c}\text { Koçyiğit et al., } \\
2016 \text { [28] }\end{array}$ & Turkey & $42.45 \pm 9.93$ & $41.77 \pm 10.5$ & $31 / 0$ & $30 / 0$ & 31 & 30 & $73.65 \pm 59.15 \mathrm{~m}$ & $69.40 \pm 40.1 \mathrm{~m}$ & 24 \\
\hline
\end{tabular}

BT, balneotherapy; con, control; NA, not available; y, years; m, months; w, weeks.

\subsection{ROBs of Included Studies}

None of the 11 studies had low ROBs (Figure 2); 6 had some concerns (unclear intended interventions [28,32], unclear intended interventions [26,29] and randomization process [30], unclear intended interventions and missing outcome data [33]), while the remaining 5 studies had high ROBs $[8,25,27,31,34]$.

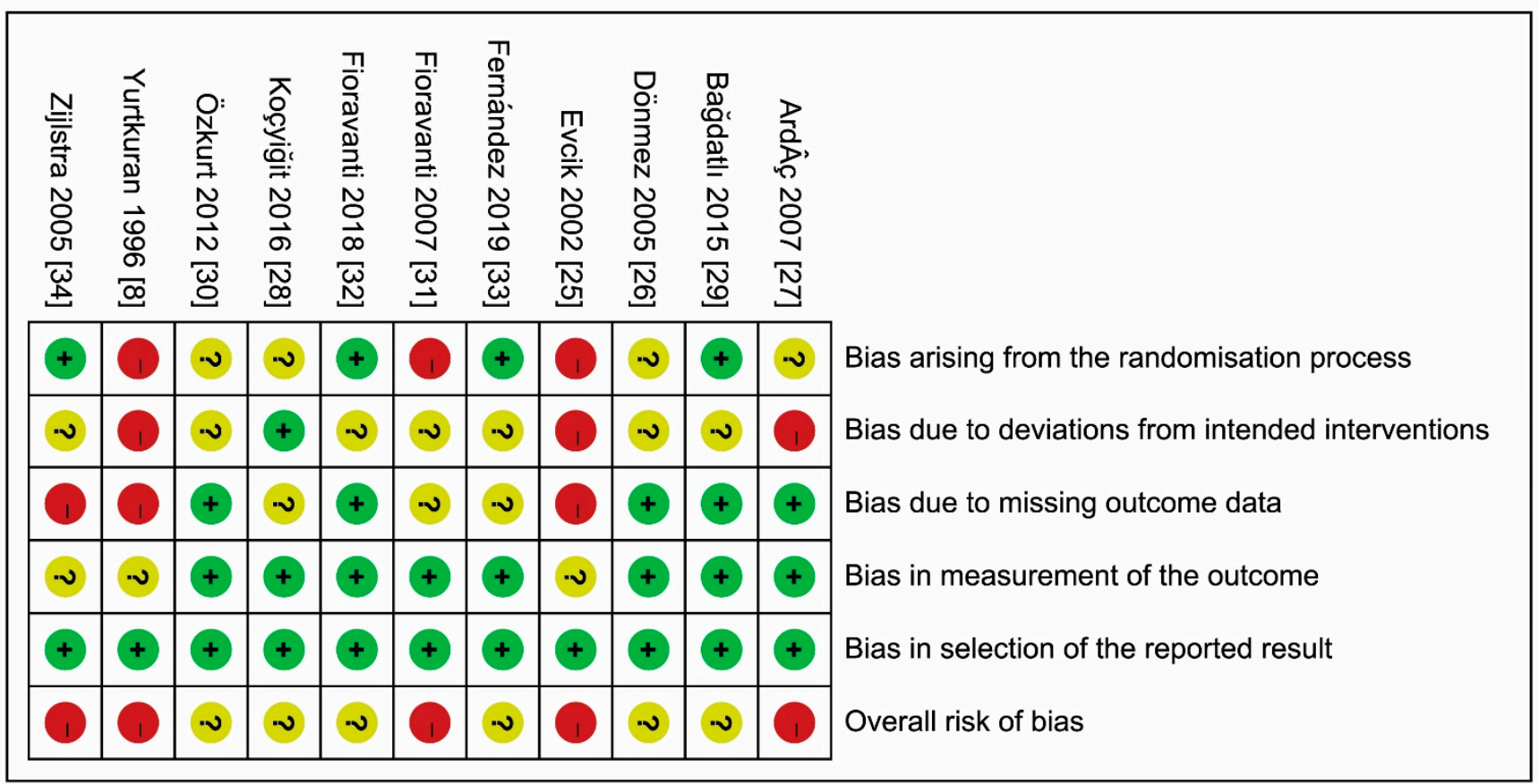

Figure 2. Risks of bias (ROBs) (2.0) within the included studies. Green circle and ' + ', low risk; red circle and '-', high risk; yellow circle and '?', unclear risk. 


\subsection{Publication Bias}

Given that the visual analysis of funnel plots (Figure 3) revealed symmetric images, the results of the meta-analysis were considered robust-to-potential publication bias (Egger's test, $p=0.66)$.

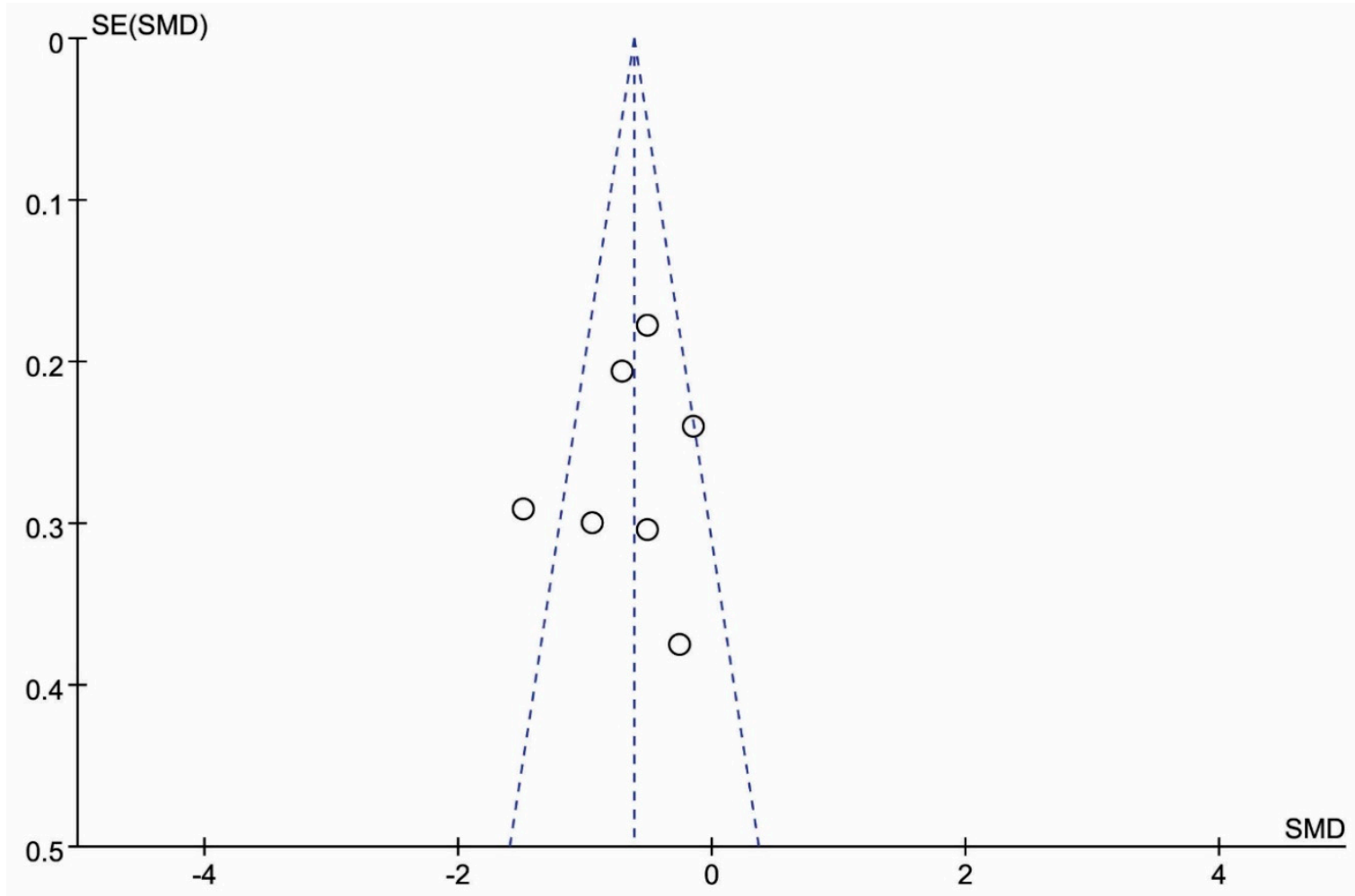

Figure 3. Funnel plot of the Fibromyalgia Impact Questionnaire (FIQ) at three months following treatment with BT. The following visual analysis of funnel plots revealed symmetric images, with robust-to-potential publication bias.

\subsection{Effects of BT Interventions}

\subsubsection{BT Intervention Sessions and Data Analysis Time Points}

Amongst 11 included studies, most BT intervention sessions were 2 weeks (8 studies), whereas 2 studies were 3 weeks, and 1 study was 2.5 weeks. A detailed list of BTs (including water temperature, therapy time, therapy session, and components) is shown in Table 3. There were nine types of BT water and seven types as control treatment. The average BT duration was $20.5 \mathrm{~min}$ (range, $15-30 \mathrm{~min}$; three types). The average BT water temperature was 36.5 (range, $34.8-38^{\circ} \mathrm{C}$; five types). There were six types of ingredients for $\mathrm{BT}$ in six studies.

For pooled analysis, we included the eight studies with outcome measures using twoweek sessions as treatment completing time point, corresponding studies with measures at three months as follow-up time point, and studies with measures at six months as final effect time point.

\subsubsection{Primary Outcome Measures Pain}

Five studies reported the comparison of pain between $\mathrm{BT}$ and control groups at two weeks as the end of BT sessions (Figure 4a). The pain scores in the BT group were $92 \%$ lower than those in the control group (SMD $=-0.92,95 \% \mathrm{CI}(-1.31$ to -0.53$), p<0.00001)$. Test statistics revealed considerable heterogeneity among these studies $\left(I^{2}=54 \%\right)$. The comparison was rated as very low quality evidence by GRADE (Table 4). 
Table 3. Detailed information and characteristics of balneotherapy.

\begin{tabular}{|c|c|c|c|c|c|c|}
\hline \multirow{2}{*}{ Studies } & \multicolumn{2}{|c|}{ Intervention } & \multirow{2}{*}{ Duration (min) } & \multirow{2}{*}{ Sessions (w) } & \multirow{2}{*}{$\begin{array}{c}\text { Temperature } \\
\left({ }^{\circ} \mathrm{C}\right)\end{array}$} & \multirow{2}{*}{ Ingredients } \\
\hline & BT & con & & & & \\
\hline $\begin{array}{c}\text { Fioravanti et al., } \\
2018 \text { [32] }\end{array}$ & Vetriolo's water & Tap water & 15 & 2 & 36 & $\begin{array}{l}\text { Highly } \\
\text { mineralized, } \\
\text { pH 5.7, sulfate, } \\
\text { calcium, } \\
\text { magnesium, } \\
\text { and iron }\end{array}$ \\
\hline $\begin{array}{l}\text { Fernández et al., } \\
2019 \text { [33] }\end{array}$ & $\begin{array}{l}\text { Bicarbonate } \\
\text { sodium water }\end{array}$ & Pharmacological & 30 & 2 & 38 & $\begin{array}{c}\text { Medium } \\
\text { mineralization, } \\
\text { alkaline, lithic, } \\
\text { fluorinated, } \\
\text { silicated }\end{array}$ \\
\hline $\begin{array}{c}\text { Dönmez et al., } \\
2005 \text { [26] }\end{array}$ & Thermal water & Pharmacological & 20 & 2 & $36 \pm 1$ & $\begin{array}{c}\text { Sodium, } \\
\text { chlorine, } \\
\text { bicarbonate and } \\
\text { fluoride }\end{array}$ \\
\hline $\begin{array}{l}\text { Özkurt et al., } \\
2012 \text { [30] }\end{array}$ & Thermal baths & Pharmacological & 20 & 2 & $36 \pm 1$ & $\begin{array}{c}\text { Sodium, } \\
\text { chloride, and } \\
\text { calcium with a } \\
\text { total } \\
\text { mineralization } \\
\text { of } 3367 \mathrm{mg} / \mathrm{L}\end{array}$ \\
\hline $\begin{array}{l}\text { Zijlstra et al., } \\
2005 \text { [34] }\end{array}$ & Seawater & NA & NA & $2^{1 / 2}$ & NA & NA \\
\hline $\begin{array}{l}\text { Bağdatlı et al., } \\
\quad 2015 \text { [29] }\end{array}$ & $\begin{array}{l}\text { Pool baths and } \\
\text { mud pack }\end{array}$ & $\begin{array}{c}\text { Exercise, } \\
\text { education, and } \\
\text { pharmacologi- } \\
\text { cal }\end{array}$ & 20 & 2 & 38 & NA \\
\hline $\begin{array}{l}\text { Evcik et al., } \\
2002[25]\end{array}$ & Thermal baths & $\begin{array}{l}\text { Pharmacological } \\
\text { and exercise }\end{array}$ & 20 & 3 & 36 & NA \\
\hline $\begin{array}{l}\text { Ardıç et al., } \\
2007 \text { [27] }\end{array}$ & $\begin{array}{l}\text { Thermal pool } \\
\text { water }\end{array}$ & $\begin{array}{l}\text { Exercise or } \\
\text { walking }\end{array}$ & 20 & 3 & 36 & NA \\
\hline $\begin{array}{c}\text { Yurtkuran et al., } \\
1996 \text { [8] }\end{array}$ & $\begin{array}{c}\text { Therapeutic } \\
\text { pool }\end{array}$ & $\begin{array}{l}\text { Relaxation } \\
\text { exercises }\end{array}$ & 20 & 2 & 37 & $\begin{array}{c}\mathrm{HCO}_{3}^{-}, \mathrm{Cl}^{-}, \\
\mathrm{F}^{-}, \mathrm{SO}_{4}^{-2}, \\
\mathrm{Ca}^{+2}, \mathrm{Mg}^{+2}, \mathrm{~K}^{+}, \\
\mathrm{Li}^{+1}\end{array}$ \\
\hline $\begin{array}{c}\text { Fioravanti et al., } \\
2007 \text { [31] }\end{array}$ & $\begin{array}{l}\text { Mud packs and } \\
\text { thermal baths }\end{array}$ & Pharmacological & 15 & 2 & $37-38$ & NA \\
\hline $\begin{array}{l}\text { Koçyiğit et al., } \\
2016 \text { [28] }\end{array}$ & $\begin{array}{c}\text { Thermomineral } \\
\text { water }\end{array}$ & Education & 20 & 4 & 34.8 & $\begin{array}{l}\mathrm{Na}, \mathrm{K}, \mathrm{Mg}, \mathrm{Ca}, \\
\mathrm{F}, \mathrm{CI}, \mathrm{Bromur}, \mathrm{I} \\
\mathrm{NO}_{2}, \mathrm{NO}_{3}, \mathrm{SO}_{4} \\
\mathrm{HCO}_{3}, \mathrm{~S}, \mathrm{HPO}_{4}\end{array}$ \\
\hline
\end{tabular}

BT, balneotherapy; con, control; w, week; NA, not available.

Six studies reported the comparison of pain between $\mathrm{BT}$ and control groups at three months (Figure $4 \mathrm{~b}$ ). BT reduced the pain score by $45 \%$ (SMD $=-0.45,95 \% \mathrm{CI}(-0.73$ to $-0.16), p=0.0002$; GRADE: moderate). Considerable heterogeneity existed among these studies $\left(I^{2}=51 \%\right)$.

Five studies reported the comparison of pain between BT and control groups at six months (Figure 4c). BT reduced the pain score by 70\% (SMD $=-0.70,95 \% \mathrm{CI}(-1.34$ to $-0.05), p=0.03$; GRADE: low). Considerable heterogeneity existed among these studies $\left(I^{2}=87 \%\right)$. 
a

\begin{tabular}{|c|c|c|c|c|c|c|c|c|c|c|c|}
\hline \multirow[b]{2}{*}{ Study or Subgroup } & \multicolumn{3}{|c|}{ BT } & \multicolumn{2}{|c|}{ Control } & \multirow[b]{2}{*}{ Total } & \multirow[b]{2}{*}{ Weight } & \multirow{2}{*}{$\begin{array}{l}\text { Std. Mean Difference } \\
\text { IV. Random. } 95 \% \mathrm{CI}\end{array}$} & \multirow{2}{*}{\multicolumn{3}{|c|}{$\begin{array}{l}\text { Std. Mean Difference } \\
\text { IV. Random. } 95 \% \mathrm{Cl}\end{array}$}} \\
\hline & Mean & SD & Total & Mean & SD & & & & & & \\
\hline Dönmez 2005 [26] & 3.78 & 2.33 & 16 & 6.08 & 2.68 & 13 & $15.1 \%$ & $-0.90[-1.67,-0.12]$ & & & \\
\hline Fioravanti 2018 [32] & 5 & 2.62 & 50 & 6.47 & 2.32 & 50 & $26.9 \%$ & $-0.59[-0.99,-0.19]$ & & & \\
\hline Koçyiğit 2016 [28] & 4.25 & 1.75 & 31 & 6.25 & 1.25 & 30 & $21.3 \%$ & $-1.29[-1.85,-0.74]$ & & & \\
\hline Yurtkuran 1996 [8] & 3.7 & 1.4 & 20 & 5.7 & 1.2 & 20 & $16.7 \%$ & $-1.50[-2.21,-0.79]$ & & & \\
\hline Özkurt 2012 [30] & 45.25 & 20.75 & 21 & 55.25 & 19.25 & 24 & $20.0 \%$ & $-0.49[-1.09,0.10]$ & & & \\
\hline Total $(95 \% \mathrm{Cl})$ & & & 138 & & & 137 & $100.0 \%$ & $-0.92[-1.31,-0.53]$ & & & \\
\hline $\begin{array}{l}\text { Heterogeneity: } \mathrm{Tau}^{2}= \\
\text { Test for overall effect: }\end{array}$ & $\begin{array}{l}0.10 ; \mathrm{Cr} \\
\mathrm{Z}=4.65\end{array}$ & $\begin{array}{l}\mathrm{i}^{2}=8.7 \\
(\mathrm{P}<0 .\end{array}$ & $\begin{array}{l}3, \mathrm{df}= \\
00001)\end{array}$ & $4(P=C$ & $.07) ; 1^{2}$ & $=54 \%$ & & & $\begin{array}{l}-1 \\
\text { Favours [BT }\end{array}$ & 0 & $\begin{array}{c}1 \\
\text { control] }\end{array}$ \\
\hline
\end{tabular}

b

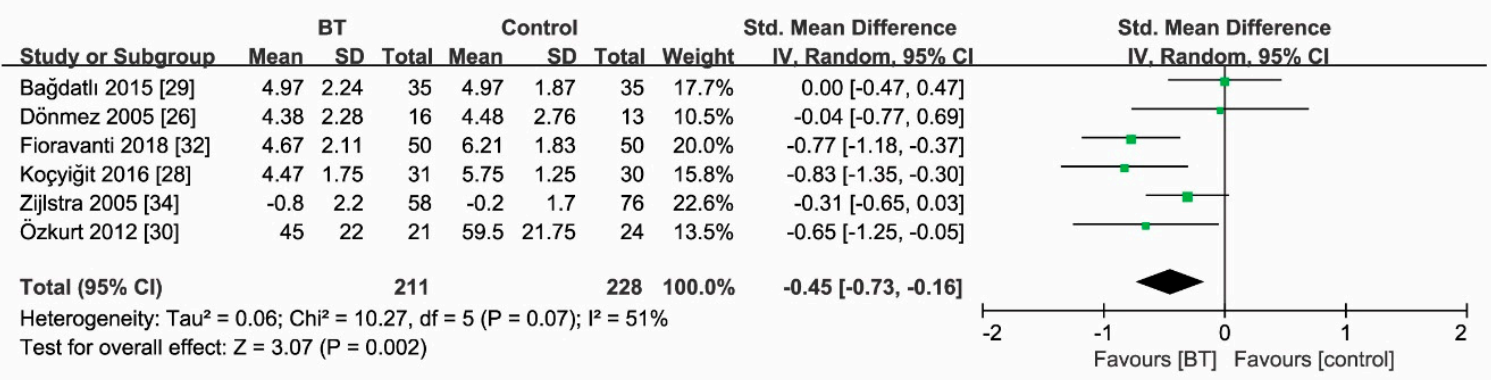

C

\begin{tabular}{|c|c|c|c|c|c|c|c|c|c|c|c|}
\hline \multirow[b]{2}{*}{ Study or Subgroup } & \multicolumn{3}{|c|}{ BT } & \multicolumn{3}{|c|}{ Control } & \multicolumn{2}{|c|}{ Std. Mean Difference } & \multirow{2}{*}{\multicolumn{2}{|c|}{$\begin{array}{l}\text { Std. Mean Difference } \\
\text { IV. Random, } 95 \% \mathrm{Cl}\end{array}$}} & \\
\hline & Mean & SD & Total & Mean & SD & Total & Weight & IV, Random, $95 \% \mathrm{Cl}$ & & & \\
\hline Dönmez 2005 [26] & 4.25 & 1.98 & 16 & 4.456 & 1.79 & 13 & $18.2 \%$ & $-0.11[-0.84,0.63]$ & & & \\
\hline Evcik 2002 [25] & 3.5 & 1 & 22 & 6.1 & 1.2 & 20 & $17.4 \%$ & $-2.32[-3.12,-1.52]$ & & & \\
\hline Fioravanti 2018 [32] & 4.96 & 2.12 & 50 & 6.36 & 2.31 & 50 & $21.7 \%$ & $-0.63[-1.03,-0.22]$ & & & \\
\hline Koçyiğit 2016 [28] & 6.75 & 1.75 & 31 & 7.75 & 0.75 & 30 & $20.5 \%$ & $-0.73[-1.25,-0.21]$ & & & \\
\hline Zijlstra 2005 [34] & 0 & 2.3 & 58 & -0.1 & 1.7 & 76 & $22.2 \%$ & $0.05[-0.29,0.39]$ & & & \\
\hline Total $(95 \% \mathrm{Cl})$ & & & 177 & & & 189 & $100.0 \%$ & $-0.70[-1.34,-0.05]$ & & & \\
\hline $\begin{array}{l}\text { Heterogeneity: } \mathrm{Tau}^{2}= \\
\text { Test for overall effect: }\end{array}$ & $\begin{array}{l}0.46 ; \mathrm{Cl} \\
=2.12\end{array}$ & $\begin{array}{l}i^{2}=3 \\
(P=\end{array}$ & $\begin{array}{l}1.97, \mathrm{df} \\
.03)\end{array}$ & $=4(\mathrm{P}$ & .000 & $01) ; 1^{2}$ & $=87 \%$ & & $\begin{array}{l}-1 \\
\text { Favours [BT] }\end{array}$ & Favours [c & $\begin{array}{l}1 \\
\text { ontrol] }\end{array}$ \\
\hline
\end{tabular}

Figure 4. Forest plot for the comparison of pain (a) at 2 weeks, showing the effect favoring balneotherapy; (b) at 3 months, showing the effect favoring balneotherapy; and (c) at 6 months, showing the effect favoring balneotherapy. Green square represent the std. mean difference, bars represent the $95 \%$ confidence interval, and black diamond represent the pooled analysis for each pain score.

Fibromyalgia Impact Questionnaire

Of the 11 RCTs, 6 used the FIQ at two weeks (Figure 5a). The mean values in Figure 5 indicated FIQ scores at the same observational time after treatment as listed for each group (BT group and control group). BT improved the FIQ score by $104 \%$ when compared with controls (SMD $=-1.04,95 \%$ CI $(-1.51$ to -0.57$), p<0.00001$; GRADE: low). Considerable heterogeneity was identified among these studies $\left(I^{2}=76 \%\right)$. 
Table 4. GRADE summary of findings.

\begin{tabular}{|c|c|c|c|c|c|c|c|c|c|c|c|c|}
\hline \multirow[b]{2}{*}{ Outcome } & \multicolumn{7}{|c|}{ Certainty Assessment } & \multicolumn{2}{|c|}{ No. of Patients } & \multicolumn{2}{|c|}{ Effect } & \multirow[b]{2}{*}{ Certainty } \\
\hline & $\begin{array}{l}\text { No. of } \\
\text { Studies }\end{array}$ & $\begin{array}{c}\text { Study } \\
\text { Design }\end{array}$ & Risk of Bias & Inconsistency & Indirectness & Imprecision & $\begin{array}{l}\text { Other Con- } \\
\text { siderations }\end{array}$ & Balneotherapy & Control & $\begin{array}{l}\text { Relative( } 95 \% \\
\text { CI) }\end{array}$ & $\begin{array}{l}\text { Absolute } \\
\text { (95\% CI) }\end{array}$ & \\
\hline $\begin{array}{c}\text { Pain at } 2 \\
\text { weeks }\end{array}$ & 5 & $\begin{array}{c}\text { Randomized } \\
\text { trials }\end{array}$ & Serious & Very serious & Not serious & Not serious & None & 138 & 137 & - & $\begin{array}{c}\text { SMD } 0.92 \text { lower } \\
\text { (1.13 lower to } 0.53 \\
\text { lower) }\end{array}$ & $\begin{array}{c}\oplus \circ \bigcirc \bigcirc \\
\text { VERY LOW }\end{array}$ \\
\hline $\begin{array}{l}\text { Pain at } 3 \\
\text { months }\end{array}$ & 6 & $\begin{array}{c}\text { Randomized } \\
\text { trials }\end{array}$ & Serious & Not serious & Not serious & Not serious & None & 211 & 228 & - & $\begin{array}{l}\text { SMD } 0.45 \text { lower } \\
\text { (0.73 lower to } 0.16 \\
\text { lower) }\end{array}$ & $\begin{array}{c}\oplus \oplus \oplus \bigcirc \\
\text { MODERATE }\end{array}$ \\
\hline $\begin{array}{l}\text { Pain at } 6 \\
\text { months }\end{array}$ & 5 & $\begin{array}{c}\text { Randomized } \\
\text { trials }\end{array}$ & Serious & Serious & Not serious & Not serious & None & 177 & 189 & - & $\begin{array}{l}\text { SMD } 0.7 \text { lower } \\
\text { (1.34 lower to } 0.05 \\
\text { lower) }\end{array}$ & $\begin{array}{l}\oplus \oplus \circ \bigcirc \\
\text { LOW }\end{array}$ \\
\hline $\begin{array}{c}\text { FIQ at } 2 \\
\text { weeks }\end{array}$ & 7 & $\begin{array}{c}\text { Randomized } \\
\text { trials }\end{array}$ & Serious & Serious & Not serious & Not serious & None & 190 & 186 & - & $\begin{array}{c}\text { SMD } 1.01 \text { lower } \\
\text { (1.43 lower to } 0.59 \\
\text { lower) }\end{array}$ & $\begin{array}{l}\oplus \oplus \circ \bigcirc \\
\text { LOW }\end{array}$ \\
\hline $\begin{array}{l}\text { FIQ at } 3 \\
\text { months }\end{array}$ & 7 & $\begin{array}{c}\text { Randomized } \\
\text { trials }\end{array}$ & Serious & Serious & Not serious & Not serious & None & 236 & 253 & - & $\begin{array}{c}\text { SMD } 0.64 \text { lower } \\
\text { (0.95 lower to } 0.33 \\
\text { lower })\end{array}$ & $\begin{array}{l}\oplus \oplus \circ \bigcirc \\
\text { LOW }\end{array}$ \\
\hline $\begin{array}{l}\text { FIQ at } 6 \\
\text { months }\end{array}$ & 4 & $\begin{array}{c}\text { Randomized } \\
\text { trials }\end{array}$ & Serious & Very serious & Not serious & Not serious & None & 155 & 169 & - & $\begin{array}{c}\text { SMD } 0.59 \text { lower } \\
\text { (0.91 lower to } 0.27 \\
\text { lower) }\end{array}$ & $\begin{array}{c}\oplus \circ 0 \bigcirc \\
\text { VERY LOW }\end{array}$ \\
\hline $\begin{array}{l}\text { TPC at } 3 \\
\text { months }\end{array}$ & 4 & $\begin{array}{c}\text { Randomized } \\
\text { trials }\end{array}$ & Serious & Not serious & Not serious & Not serious & None & 126 & 143 & - & $\begin{array}{c}\text { SMD } 0.47 \text { lower } \\
\text { (0.71 lower to } 0.22 \\
\text { lower })\end{array}$ & $\begin{array}{c}\oplus \oplus \oplus \bigcirc \\
\text { MODERATE }\end{array}$ \\
\hline $\begin{array}{l}\text { TPC at } 6 \\
\text { months }\end{array}$ & 4 & $\begin{array}{c}\text { Randomized } \\
\text { trials }\end{array}$ & Serious & Very serious & Not serious & Not serious & None & 127 & 139 & - & $\begin{array}{l}\text { SMD } 0.89 \text { lower } \\
\text { (1.85 lower to } 0.07 \\
\text { higher) }\end{array}$ & $\begin{array}{c}\oplus 00 \bigcirc \\
\text { VERY LOW }\end{array}$ \\
\hline $\begin{array}{l}\text { BDI at } 2 \\
\text { weeks }\end{array}$ & 4 & $\begin{array}{c}\text { Randomized } \\
\text { trials }\end{array}$ & Serious & Not serious & Not serious & Not serious & None & 84 & 81 & - & $\begin{array}{l}\text { SMD } 0.32 \text { lower } \\
\text { (0.63 lower to } 0.01 \\
\text { lower })\end{array}$ & $\begin{array}{c}\oplus \oplus \oplus \bigcirc \\
\text { MODERATE }\end{array}$ \\
\hline $\begin{array}{l}\text { BDI at } 3 \\
\text { months }\end{array}$ & 4 & $\begin{array}{c}\text { Randomized } \\
\text { trials }\end{array}$ & Serious & Serious & Not serious & Not serious & None & 133 & 148 & - & $\begin{array}{c}\text { SMD } 0.23 \text { lower } \\
\text { (0.64 lower to } 0.17 \\
\text { higher) }\end{array}$ & $\begin{array}{l}\oplus \oplus \circ \bigcirc \\
\text { LOW }\end{array}$ \\
\hline $\begin{array}{l}\text { BDI at } 6 \\
\text { months }\end{array}$ & 3 & $\begin{array}{c}\text { Randomized } \\
\text { trials }\end{array}$ & Serious & Not serious & Not serious & Not serious & None & 96 & 109 & - & $\begin{array}{c}\text { SMD } 0.45 \text { lower } \\
\text { (0.73 lower to } 0.17 \\
\text { lower) }\end{array}$ & $\begin{array}{c}\oplus \oplus \oplus \bigcirc \\
\text { MODERATE }\end{array}$ \\
\hline
\end{tabular}

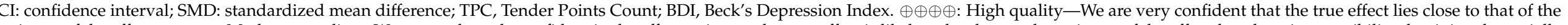

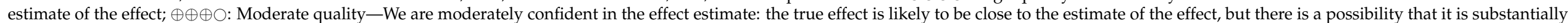

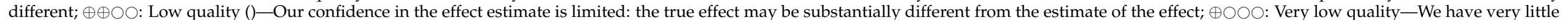
confidence in the effect estimate: the true effect is likely to be substantially different from the estimate of effect 
a

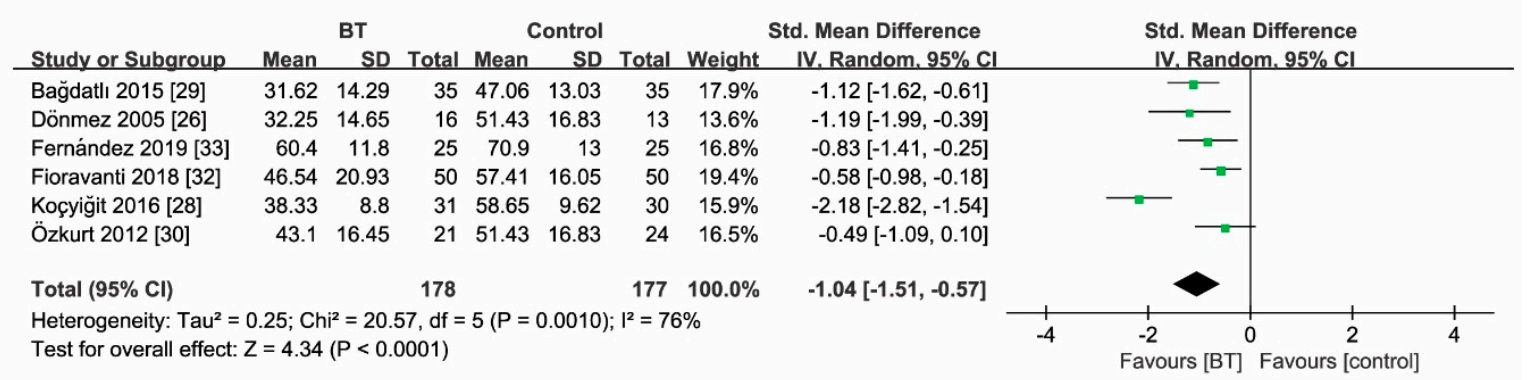

b

\begin{tabular}{|c|c|c|c|c|c|c|c|c|c|c|c|c|}
\hline \multirow[b]{2}{*}{ Study or Subgroup } & \multicolumn{3}{|c|}{ BT } & \multicolumn{2}{|c|}{ Control } & \multicolumn{3}{|r|}{ Std. Mean Difference } & \multirow{2}{*}{\multicolumn{4}{|c|}{$\begin{array}{l}\text { Std. Mean Difference } \\
\text { IV. Random. } 95 \% \mathrm{Cl}\end{array}$}} \\
\hline & Mean & SD & Total & Mean & SD & Total & Weight & IV. Random. $95 \% \mathrm{Cl}$ & & & & \\
\hline Bağdatı 2015 [29] & 40.18 & 14.46 & 35 & 42.34 & 14.24 & 35 & $15.5 \%$ & $-0.15[-0.62,0.32]$ & & & & \\
\hline Dönmez 2005 [26] & 36.73 & 13.78 & 16 & 40.58 & 17.02 & 13 & $10.2 \%$ & $-0.24[-0.98,0.49]$ & & & & \\
\hline Fernández 2019 [33] & 64.4 & 10.7 & 25 & 75 & 11.4 & 25 & $12.9 \%$ & $-0.94[-1.53,-0.36]$ & & & & \\
\hline Fioravanti 2018 [32] & 43.03 & 18.81 & 50 & 54.98 & 14.99 & 50 & $17.1 \%$ & $-0.70[-1.10,-0.29]$ & & & & \\
\hline Koçyiğit 2016 [28] & 39.17 & 10.08 & 31 & 54.27 & 9.97 & 30 & $13.2 \%$ & $-1.49[-2.06,-0.92]$ & & & & \\
\hline Zijlstra 2005 [34] & -6.3 & 11.4 & 58 & -0.9 & 10.2 & 76 & $18.5 \%$ & $-0.50[-0.85,-0.15]$ & & & & \\
\hline Özkurt 2012 [30] & 40.8 & 16 & 21 & 49.4 & 16.9 & 24 & $12.7 \%$ & $-0.51[-1.11,0.08]$ & & & & \\
\hline Total $(95 \% \mathrm{Cl})$ & & & 236 & & & 253 & $100.0 \%$ & $-0.64[-0.95,-0.33]$ & & & & \\
\hline \multicolumn{9}{|c|}{$\begin{array}{l}\text { Heterogeneity: } \mathrm{Tau}^{2}=0.10 ; \mathrm{Chi}^{2}=15.61, \mathrm{df}=6(P=0.02) ; I^{2}=62 \% \\
\text { Test for overall effect: } Z=4.08(P<0.0001)\end{array}$} & $\begin{array}{lcc}-4 & -2 & 0 \\
\text { Favours [experimental] }\end{array}$ & $\begin{array}{lr}0 & 2 \\
& \text { Favours [ce }\end{array}$ & $\begin{array}{l}2 \\
\text { control] }\end{array}$ & 4 \\
\hline
\end{tabular}

C

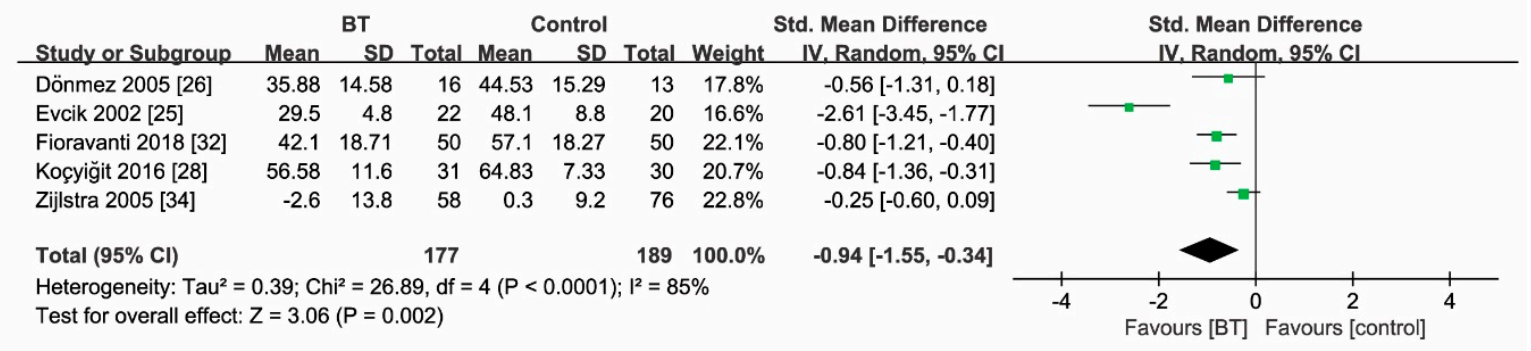

Figure 5. Forest plot (a) for the comparison of the FIQ score at 2 weeks, showing the effect favoring balneotherapy; (b) at 3 months, showing the effect favoring balneotherapy; and (c) at 6 months, showing the effect favoring balneotherapy. In the figure, the mean indicates mean FIQ values. Green square represent the std. mean difference, bars represent the $95 \%$ confidence interval, and black diamond represent the pooled analysis for each FIQ score.

Seven studies reported the comparison of the FIQ score between BT and control groups at three months (Figure $5 b$ ). BT improved the FIQ score by $64 \%$ (SMD $=-0.64,95 \%$ CI $(-0.95$ to -0.33$), p<0.00001$; GRADE: low). Considerable heterogeneity existed among these studies $\left(I^{2}=62 \%\right)$.

Five studies reported the comparison of the FIQ score between BT and control groups at six months (Figure 5c). Analogously, pooled results of subgroup analysis indicated that BT improved the FIQ score by $94 \%$ when compared with controls (SMD $=-0.94,95 \%$ CI ( -1.55 to -0.34$), p=0.002$; GRADE: very low). Considerable heterogeneity presented among these studies $\left(I^{2}=85 \%\right)$.

Tender Points Count

Four studies measured the TPC as the outcome at two weeks (Figure 6a). The mean values in Figure 6 indicated the TPC at the same observational time after treatment as listed for each group (BT group and control group). BT improved the clinical efficacy of the TPC by $94 \%$ when compared with controls (SMD $=-0.94,95 \% \mathrm{CI}(-1.69$ to -0.18$), p=0.02$; GRADE: very low). Considerable heterogeneity existed among these studies $\left(I^{2}=81 \%\right)$. 
a

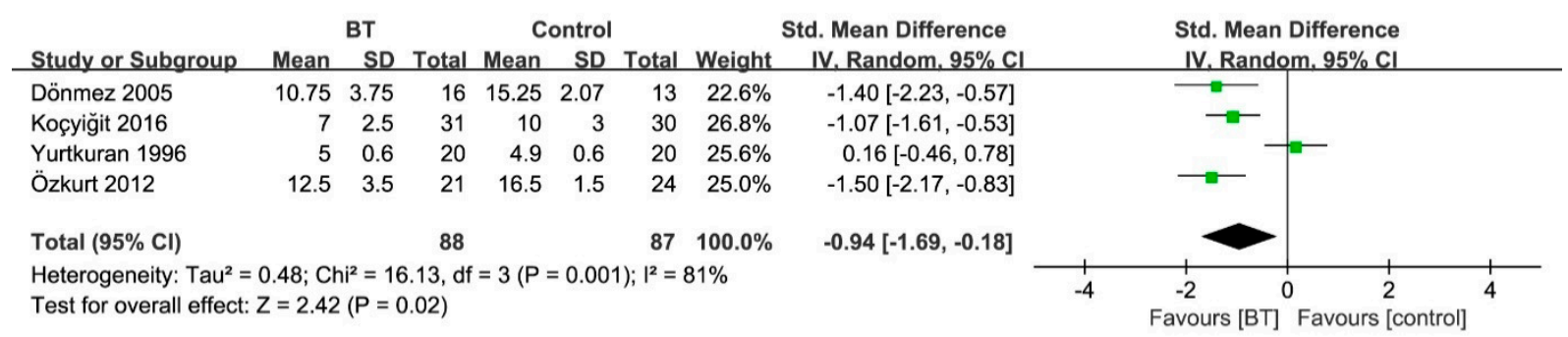

b

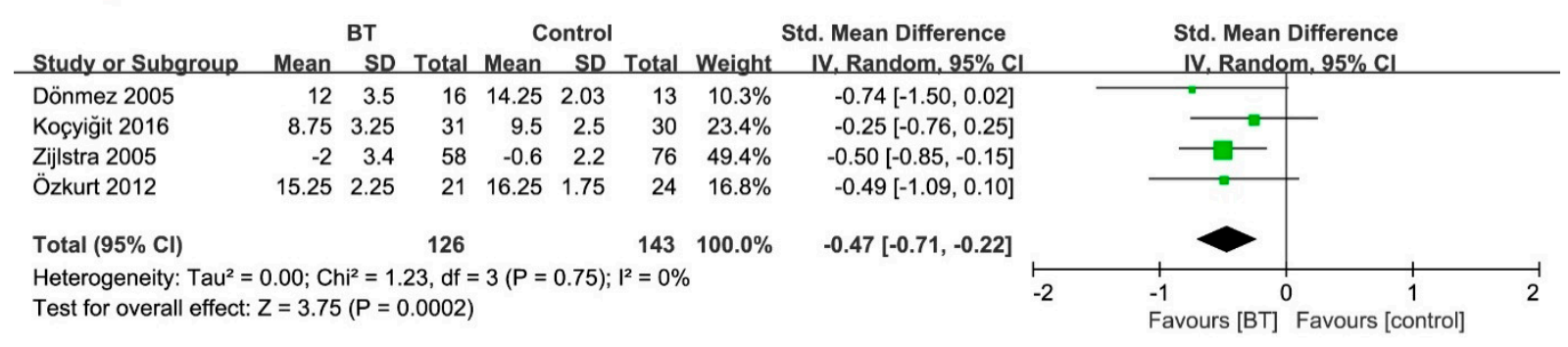

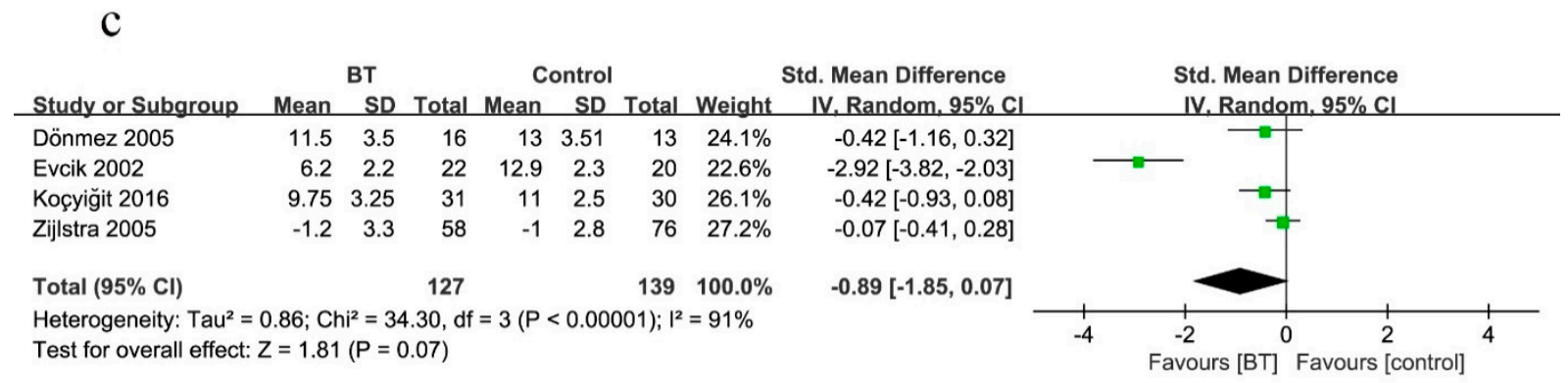

Figure 6. Forest plot for the comparison of the TPC (a) at 2 weeks, showing the effect favoring balneotherapy; (b) at 3 months, showing the effect favoring balneotherapy; and (c) at 6 months, showing the effect not favoring balneotherapy. In the figure, the mean indicates mean TPC values. Green square represent the std. mean difference, bars represent the $95 \%$ confidence interval, and black diamond represent the pooled analysis for each TPC.

Four studies measured the TPC as the outcome at three months (Figure 6b). BT improved the clinical efficacy of the TPC by $47 \%$ when compared with controls (SMD $=-0.47$, $95 \% \mathrm{CI}(-0.71$ to -0.22$), p=0.0002 ;$ GRADE: moderate). No heterogeneity existed ( $p=0.75$, $\left.I^{2}=0 \%\right)$.

Four studies reported the comparison of the TPC between BT and control groups at six months (Figure 6c). There was no statistical difference effect on the TPC (SMD $=-0.89$, $95 \% \mathrm{CI}(-1.85$ to 0.07$), p=0.07$; GRADE: very low). Considerable heterogeneity was noted among these studies $\left(I^{2}=91 \%\right)$.

\section{Beck's Depression Index}

Three studies measured the BDI as the outcome at two weeks (Figure 7a). There was no statistical difference effect on the BDI (SMD $=-0.35,95 \%$ CI ( -0.73 to 0.04$), p=0.06$; GRADE: moderate), with no heterogeneity $\left(p=0.27, I^{2}=24 \%\right)$. 


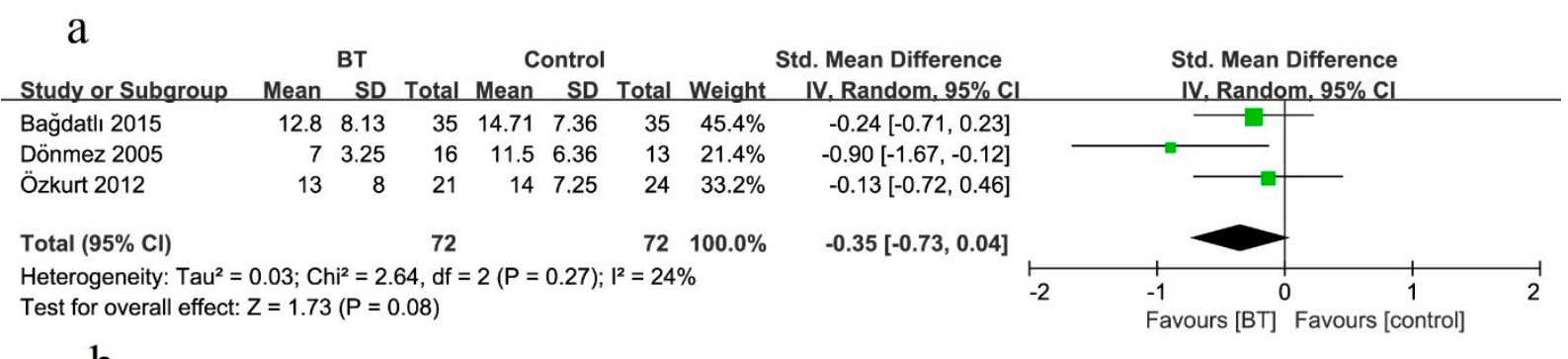

b

\begin{tabular}{|c|c|c|c|c|c|c|c|c|c|c|c|}
\hline \multirow[b]{2}{*}{ Study or Subgroup } & \multicolumn{3}{|c|}{ BT } & \multicolumn{3}{|c|}{ Control } & \multicolumn{2}{|r|}{ Std. Mean Difference } & \multirow{2}{*}{\multicolumn{2}{|c|}{$\begin{array}{l}\text { Std. Mean Difference } \\
\text { IV. Random, } 95 \% \mathrm{Cl}\end{array}$}} & \\
\hline & Mean & SD & Total & Mean & SD & Total & Weight & IV. Random, $95 \% \mathrm{Cl}$ & & & \\
\hline Bağdatlı 2015 & 11.97 & 8.06 & 35 & 11.31 & 5.42 & 35 & $27.1 \%$ & $0.10[-0.37,0.56]$ & & + & \\
\hline Dönmez 2005 & 11.5 & 5.5 & 16 & 10 & 6.07 & 13 & $17.7 \%$ & $0.25[-0.48,0.99]$ & & & \\
\hline Zijlstra 2005 & -1.7 & 5.5 & 58 & 1.2 & 5.3 & 76 & $32.4 \%$ & $-0.54[-0.88,-0.19]$ & & & \\
\hline Özkurt 2012 & 10 & 5 & 24 & 13.25 & 6 & 24 & $22.8 \%$ & $-0.58[-1.16,-0.00]$ & & & \\
\hline Total $(95 \% \mathrm{Cl})$ & & & 133 & & & 148 & $100.0 \%$ & $-0.23[-0.64,0.17]$ & & & \\
\hline \multicolumn{9}{|c|}{$\begin{array}{l}\text { Heterogeneity: } \mathrm{Tau}^{2}=0.10 ; \mathrm{Ch}^{2}=7.55, \mathrm{df}=3(P=0.06) ;\left.\right|^{2}=60 \% \\
\text { Test for overall effect: } Z=1.14(P=0.25)\end{array}$} & $\begin{array}{l}-1 \\
\text { Favours [BT] }\end{array}$ & Favours [c & $\begin{array}{l}1 \\
\text { control] }\end{array}$ \\
\hline
\end{tabular}

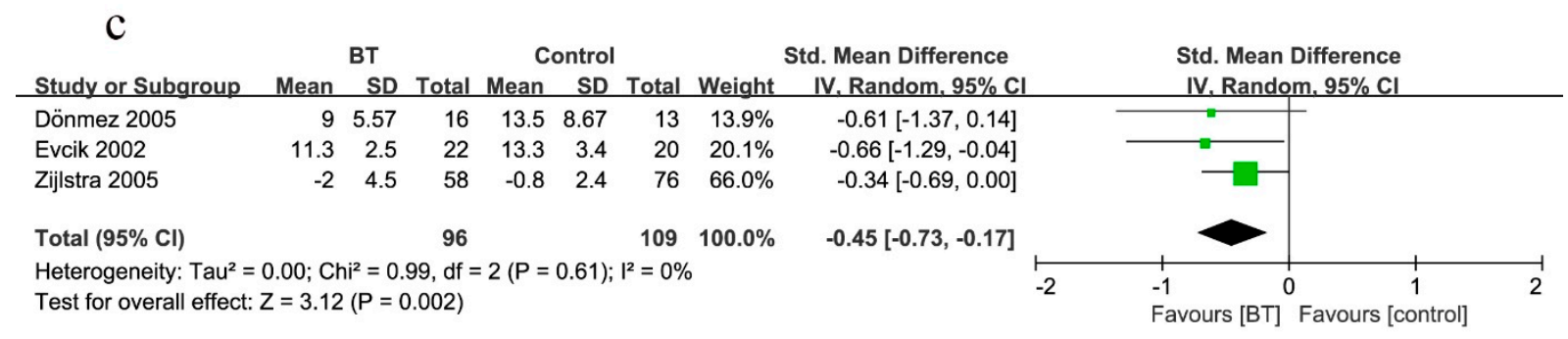

Figure 7. Forest plot for the comparison of the BDI (a) at 2 weeks, showing the effect not favoring balneotherapy; (b) at 3 months, showing the effect not favoring balneotherapy; and (c) at 6 months, showing the effect favoring balneotherapy. Green square represent the std. mean difference, bars represent the $95 \%$ confidence interval, and black diamond represent the pooled analysis for each BDI.

Four studies measured the BDI as the outcome at three months (Figure $7 \mathrm{~b}$ ). Analogously, there was no statistical difference effect on the BDI (SMD $=-0.23,95 \% \mathrm{CI}(-0.64$ to $0.17), p=0.25$; GRADE: low). Considerable heterogeneity was noted among these studies $\left(I^{2}=60 \%\right)$.

Three studies reported the comparison of the BDI between BT and control groups at six months (Figure 7c). BT improved the BDI by $45 \%$ when compared with controls at the end of treatment (SMD $=-0.45,95 \% \mathrm{CI}(-0.73$ to -0.17$), p=0.002$; GRADE: moderate), with no heterogeneity $\left(p=0.61, I^{2}=0 \%\right)$.

\subsection{Sensitivity Analyses}

Sensitivity analyses according to sample size $(n \leq 25,>25)$ revealed unaltered outcome measures as the FIQ score at two weeks and three months after treatment (Figures 8 and 9). Moreover, statistical heterogeneity of analysis for the effect size of the FIQ score in two weeks $\left(I^{2}=71 \%\right)$ and three months $\left(I^{2}=62 \%\right)$ substantially decreased (two weeks: $I^{2}=0 \%$; three months: $I^{2}=12 \%$ ) by removing the study of Koçyiğit et al. The magnitude of the effect size decreased (two weeks: SMD $=0.78 ; 95 \%$ CI $(-1.01,-0.55), p<0.00001$; three months: SMD $=0.52 ; 95 \%$ CI $(-0.73,-0.31), p<0.00001)$. 


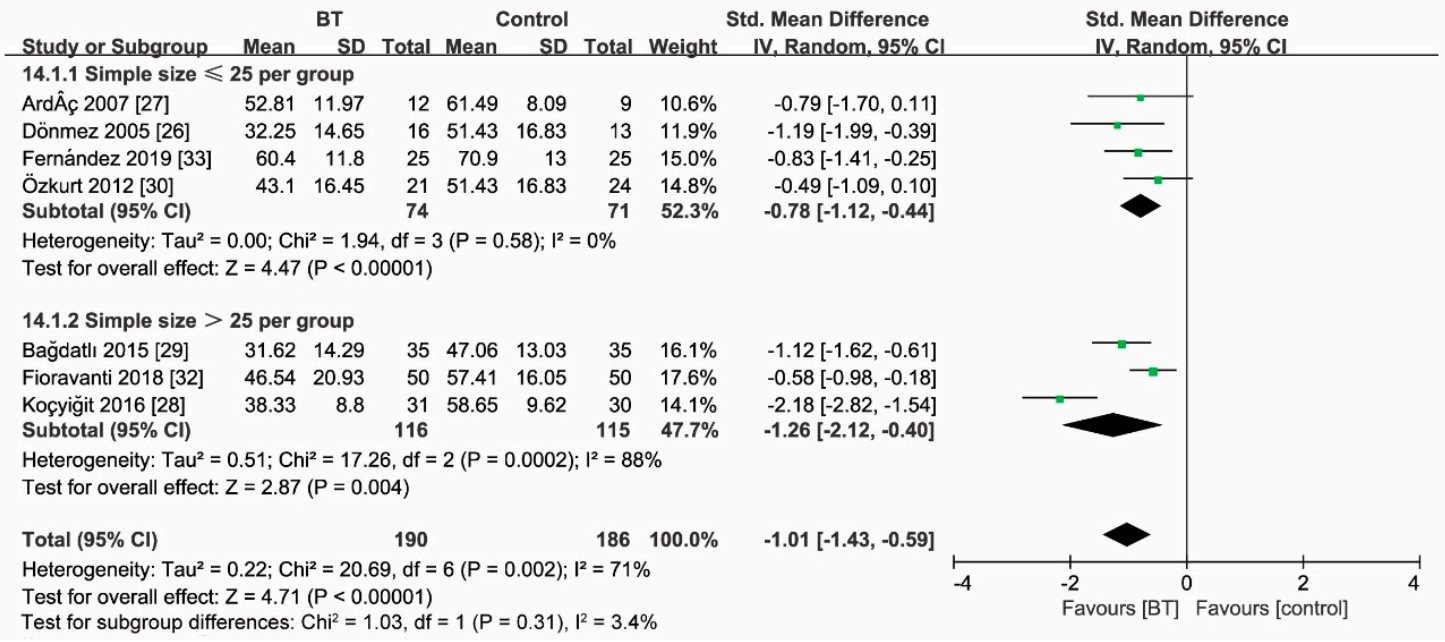

Figure 8. Sensitivity analysis for the sample size (FIQ at 2 weeks), indicating unfaltering FIQ efficacy at 2 weeks after treatment. Green square represent the std. mean difference, bars represent the $95 \%$ confidence interval, and black diamond represent the pooled analysis for FIQ score at 2 weeks in different simple size group.

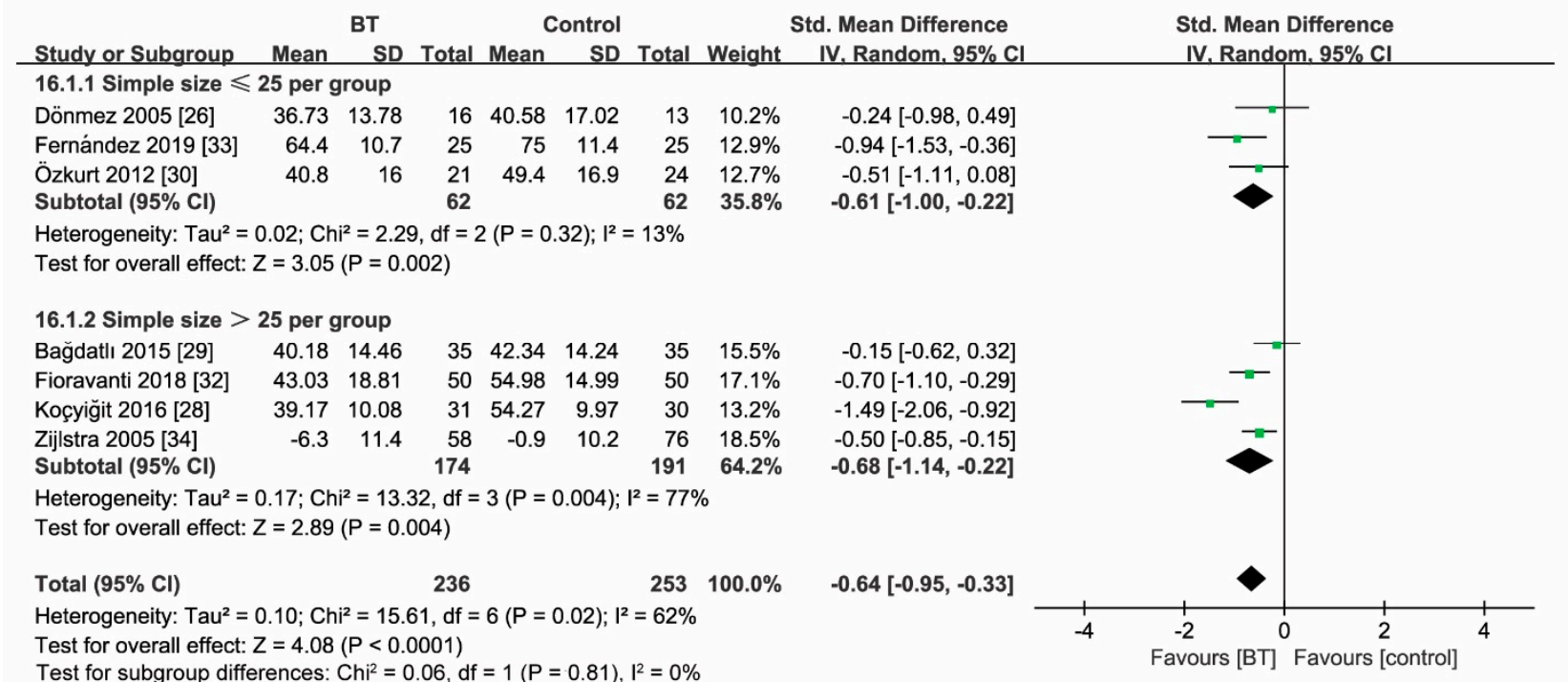

Figure 9. Sensitivity analysis for the sample size (FIQ at 3 months), showing unfaltering FIQ efficacy at 3 months after treatment. Green square represent the std. mean difference, bars represent the $95 \%$ confidence interval, and black diamond represent the pooled analysis for FIQ score at 3 months in different simple size group.

\section{Discussion}

The systematic review and meta-analysis first addressed the effect of BT on FMS at triple time points with a clear level of evidence reflected by GRADE. Amongst 672 participants, very low to moderate evidence indicates that BT can benefit FMS in pain and quality-of-life improvement, whereas tenderness and depression improvement is uncertain at different time phases.

Although guidelines or recommendations are available for the management of FMS, they are mostly based on expert consensus with some limitations [35]. European League Against Rheumatism (EULAR) recommendations for the management of FMS [5] were updated in 2017 based on systematic reviews. The importance of the multidisciplinary approach was highlighted, with special emphasis on non-pharmacological treatments for FMS. It should be stressed that BT was largely neglected in the EULAR recommendations for FMS, which used the term hydrotherapy/spa therapy. According to the nomenclature 
as a MeSH term, BT is not equal to or the same as hydrotherapy or spa therapy. Neumann's study [12] is the only available meta-analysis on BT in the EULAR recommendations, covering the literature up to April 2013. Six RCTs with 311 participants were analyzed for their meta-analysis, including 149 patients in the BT group and 162 individuals in the control group. Without a GRADE basis, they concluded moderate evidence of a medium-tolarge effect on pain and the TPC for BT, a medium effect on the FIQ score, and no significant effect on the BDI. Importantly, outcome measures were ambiguous in that meta-analysis. All outcomes were analyzed in the final treatment and at follow-up, but the time frames were not available. In fact, the treatment sessions of BT were 2.5 weeks in Zijlstra [34] and 3 weeks in Evcik [25] and Ardıç [27]. Zijlstra et al. [34] provided Visual Analogue Scale (VAS) pain data at 3,6, and 12 months after treatment. Only the data of six months of follow-up were included in their study [12]. Moreover, the TPC and FIQ data at three months were included in the previous study [12]. Thereafter, there have been no latest systematic reviews regarding the topic during the past six years.

This meta-analysis provided recent evidence for the potential treatment of FMS, including 11 selected studies with a total of 672 participants. The updated four studies greatly expanded the number of participants, significantly improving the reliability of the meta-analysis results. Moreover, we extracted all informative data from original RCTs, which may significantly improve reliability. The time points for the extracted data set were at two weeks after the start of treatment, three months, and six months as the final treatment.

Our findings are consistent with previous meta-analyses or reviews [12,13,15]. Notably, we found very low and moderate evidence that BT can benefit FMS with the TPC at two weeks and three months, but there is no significant effect at six months. This point is contrary to a previous meta-analysis [12] or the others where authors neglected the data on the TPC at follow-up (three months) [30].

Three included studies adopted the $100 \mathrm{~mm}$ Visual Analog Scale [26,30,31]. A significant improvement of pain connected with FMS was registered. The degrees of pain relief vary among the included studies, with different baselines. Functional capacity in daily-living activities were evaluated by the FIQ [36]. The results of this meta-analysis showed that BT can significantly improve functional outcomes. Interestingly, the efficacy of BT decreased with long-lasting treatment, although it was still effective in the last follow-up (24 weeks). However, this interesting finding was not observed in Altan's study [37]. Probably, this was due to the absence of subsequent treatment after BT able to maintain persistent efficacy. Similarly, the current study found that the TPC significantly reduced in the bathing treatment group in comparison with the control group. There was no significant effect on the TPC at six months. This finding is also consistent with previous studies [38,39], which demonstrated that BT has a significant effect on the improvement of the TPC. It is possible that the function of BT in the elimination of inflammatory factors reached an extreme in a certain period of treatment time [40]. Consequently, FMS patients can still choose BT as an early treatment option. Beyond that time, the pain relief from BT cannot continue. Therefore, the FMS patient's adherence to longer treatment is needed to continue the effect [41], and maybe intermittent safe administration of non-steroidal anti-inflammatory drugs would help [42]. Improvement in mental health was less pronounced and quick in physical health, suggesting that BT exerts predominantly physical effects. As pain and the quality of life improve, depression slowly improves.

The underlying mechanisms of BT effects can be due to heat, mineral content, and other physiologic and endocrine effects $[43,44]$. Thermal stress stimulation exerts analgesic effects on nerve endings by increasing the pain threshold. It alleviates muscle spasms and activates the pain-relieving inhibition system through the gamma fibers of muscle spindles. According to gate theory, pain relief may be caused by water temperature and pressure on the skin $[45,46]$. Physiologically, heat application leads to increased blood circulation, and heat application to inflamed tissue induces oxygen free radical removal and enhances the repair of the inflammatory tissue [47-49]. In different musculoskeletal diseases, the effect 
of BT on pain and function is significantly better and longer than that of a tap water bath at the same temperature [50]. The minerals that are dissolved in the water play an important part in the mechanism of action of BT. However, there have been no established criteria for these important parameters regarding BT for FMS.

High heterogeneity was noted between included studies due to several factors. First, observational phases varied, as listed in Table 2. The last follow-up time after intervention is an important factor in determining the sustained effectiveness of BT. Notably, exposure times and temperature for BT varied. Second, the composition of the mineral water and the place of spa therapy were different. Third, the definition of quality of life was not consistent due to different economic levels and regional cultures. Finally, due to the special hallmarks of the intervention, almost all included randomized controlled studies were not perfect in terms of the blind methodology. These factors may be contributive to the inter-group differences, affecting the entire heterogeneity of included studies.

This meta-analysis had several limitations. The sample sizes in the included studies were small, and this was a confusing bias. Moreover, there were no unified evidence-based diagnostic regimens for included studies, including mechanisms, common characteristics, and comorbidities, capable of improving the recognition of FMS in clinical practice. Therefore, we tried to choose randomized control trials based on FMS guidelines. Furthermore, this meta-analysis including 11 RCTs could not identify the underlying factors leading to heterogeneity. Additional studies to perform subgroup analyses are needed in the future.

\section{Conclusions}

This meta-analysis highlights there is, in fact, a role that BT can and should play in the complex treatment of FMS. In patients dealing with FMS, any opportunity to improve daily functionality and reduce chronic pain is a welcome addition to the treatment regimen. We hope this study will propagate further discussion with the consideration of the implementation of BT in the treatment for patients with FMS. The meta-analysis also brings to light that there is a clear need for further delineation of the duration and subtypes of BT, as the current data assessing this are limited. Larger-sample RCTs with similar treatment criteria, a longer follow-up time, and established evaluation criteria are needed.

Author Contributions: H.-Q.W. had full access to all of the data in the study and takes responsibility for the integrity of the data and the accuracy of the data analysis. Conceptualization, C.-F.C. and H.-Q.W.; Methodology, K.-L.M., Q.-B.W. and G.V.; Software, C.-F.C. and Q.-L.L.; Data curation, C.F.C., F.-J.L., and M.-H.Z.; Writing-original, C.-F.C. and K.-L.M.; Drafting of the manuscript, H.-Q.W., O.V., and D.M.; Administrative, technical, or material support, H.-Q.W. and G.V. All authors have read and agreed to the published version of the manuscript.

Funding: The authors disclosed receipt of the following financial support for the research, authorship, and/or publication of this article: This work was supported by the Basic and Frontier Research Projects of Chongqing, China (grant numbers Ycstc, 2017nc5); the Chongqing Municipal Commission of Health and Family Planning (grant number 2017MSXM081); and the National Natural Science Foundation of China (grant number 81572182).

Institutional Review Board Statement: Ethical review approval were waived for this study, due the study is based on previously conducted studies and does not contain any studies with human participants or animals made by the authors without previous approval.

Informed Consent Statement: Not applicable.

Data Availability Statement: The data presented in this study are available in Figures 4-9 and Tables 2 and 3.

Acknowledgments: We thank all the researchers and participants involved in the included studies. We also thank the Paolo Procacci Foundation for its support in the editing and publishing processes.

Conflicts of Interest: The authors declare no conflict of interest. 


\section{References}

1. Clauw, D.J. Fibromyalgia: A clinical review. JAMA 2014, 311, 1547-1555. [CrossRef] [PubMed]

2. Wolfe, F.; Brahler, E.; Hinz, A.; Hauser, W. Fibromyalgia prevalence, somatic symptom reporting, and the dimensionality of polysymptomatic distress: Results from a survey of the general population. Arthr. Care Res. 2013, 65, 777-785. [CrossRef] [PubMed]

3. Del Giorno, R.; Skaper, S.; Paladini, A.; Varrassi, G.; Coaccioli, S. Palmitoylethanolamide in Fibromyalgia: Results from Prospective and Retrospective Observational Studies. Pain Ther. 2015, 4, 169-178. [CrossRef] [PubMed]

4. McVeigh, J.G.; McGaughey, H.; Hall, M.; Kane, P. The effectiveness of hydrotherapy in the management of fibromyalgia syndrome: A systematic review. Rheumatol. Int. 2008, 29, 119-130. [CrossRef]

5. Macfarlane, G.J.; Kronisch, C.; Dean, L.E.; Atzeni, F.; Häuser, W.; Fluß, E.; Choy, E.; Kosek, E.; Amris, K.; Branco, J.; et al. EULAR revised recommendations for the management of fibromyalgia. Ann. Rheum. Dis. 2016, 76, 318-328. [CrossRef]

6. Miyamoto, G.C.; Lin, C.-W.C.; Cabral, C.M.N.; Van Dongen, J.M.; Van Tulder, M.W. Cost-effectiveness of exercise therapy in the treatment of non-specific neck pain and low back pain: A systematic review with meta-analysis. Br. J. Sports Med. 2018, 53, 172-181. [CrossRef]

7. Arnold, L.M.; Bennett, R.M.; Crofford, L.J.; Dean, L.E.; Clauw, D.J.; Goldenberg, D.L.; Fitzcharles, M.-A.; Paiva, E.S.; Staud, R.; Sarzi-Puttini, P.; et al. AAPT Diagnostic Criteria for Fibromyalgia. J. Pain 2019, 20, 611-628. [CrossRef]

8. Yurtkuran, M.; Celiktas, M. A randomized, controlled trial of balneotherapy in the treatment of patients with primary fibromyalgia syndrome. Phys. Med. Rehabil. Kurortmed. 1996, 6, 109-112. [CrossRef]

9. Sukenik, S.; Flusser, D.; Abu-Shakra, M. The Role of Spa Therapy in Various Rheumatic Diseases. Rheum. Dis. Clin. N. Am. 1999, 25, 883-897. [CrossRef]

10. Eroksuz, R.; Erol Forestier, F.B.; Karaaslan, F.; Forestier, R.; Issever, H.; Erdogan, N.; Karagulle, M.Z.; Donmez, A. Comparison of intermittent and consecutive balneological outpatient treatment (hydrotherapy and peloidotherapy) in fibromyalgia syndrome: A randomized, single-blind, pilot study. Int. J. Biometeorol. 2020, 64, 513-520. [CrossRef]

11. Rapoliene, L.; Razbadauskas, A.; Mockeviciene, D.; Varzaityte, L.; Skarbaliene, A. Balneotherapy for musculoskeletal pain: Does the mineral content matter? Int. J. Biometeorol. 2020, 64, 965-979. [CrossRef] [PubMed]

12. Naumann, J.; Sadaghiani, C. Therapeutic benefit of balneotherapy and hydrotherapy in the management of fibromyalgia syndrome: A qualitative systematic review and meta-analysis of randomized controlled trials. Arthr. Res. Ther. 2014, 16, R141. [CrossRef]

13. Fraioli, A.; Grassi, M.; Mennuni, G.; Geraci, A.; Petraccia, L.; Fontana, M.; Conte, S.; Serio, A. Clinical researches on the efficacy of spa therapy in fibromyalgia. A systematic review. Ann. Dell'Ist. Super. Sanità 2013, 49, 219-229.

14. Verhagen, A.P.; de Vet, H.C.; de Bie, R.A.; Kessels, A.G.; Boers, M.; Knipschild, P.G. Balneotherapy for rheumatoid arthritis and osteoarthritis. Cochrane Database Syst. Rev. 2000. [CrossRef]

15. Guidelli, G.M.; Tenti, S.; De Nobili, E.; Fioravanti, A. Fibromyalgia Syndrome and Spa Therapy: Myth or Reality? Clin. Med. Insights Arthr. Musculoskelet. Disord. 2012, 5, 19-26. [CrossRef] [PubMed]

16. Moher, D.; Liberati, A.; Tetzlaff, J.; Altman, D.G. Preferred reporting items for systematic reviews and meta-analyses: The PRISMA statement. BMJ 2009, 339, b2535. [CrossRef]

17. Wolfe, F.; Smythe, H.A.; Yunus, M.B.; Bennett, R.M.; Bombardier, C.; Goldenberg, D.L.; Tugwell, P.; Campbell, S.M.; Abeles, M.; Clark, P.; et al. The American College of Rheumatology 1990 Criteria for the Classification of Fibromyalgia. Report of the Multicenter Criteria Committee. Arthr. Rheum. 1990, 33, 160-172. [CrossRef]

18. Wolfe, F.; Clauw, D.J.; Fitzcharles, M.A.; Goldenberg, D.L.; Katz, R.S.; Mease, P.; Russell, A.S.; Russell, I.J.; Winfield, J.B.; Yunus, M.B. The American College of Rheumatology preliminary diagnostic criteria for fibromyalgia and measurement of symptom severity. Arthr. Care Res. 2010, 62, 600-610. [CrossRef]

19. Hozo, S.P.; Djulbegovic, B.; Hozo, I. Estimating the mean and variance from the median, range, and the size of a sample. BMC Med. Res. Methodol. 2005, 5, 13. [CrossRef]

20. Sterne, J.A.C.; Savović, J.; Page, M.J.; Elbers, R.G.; Blencowe, N.S.; Boutron, I.; Cates, C.J.; Cheng, H.-Y.; Corbett, M.S.; Eldridge, S.M.; et al. RoB 2: A revised tool for assessing risk of bias in randomised trials. BMJ 2019, 366, 14898. [CrossRef]

21. Puhan, A.M.; Schünemann, H.J.; Murad, M.H.; Li, T.; Brignardello-Petersen, R.; A Singh, J.; Kessels, A.G.; Guyatt, G.H. A GRADE Working Group approach for rating the quality of treatment effect estimates from network meta-analysis. BMJ 2014, 349 , g5630. [CrossRef]

22. Deeks, J.J.; Higgins, J.P.T.; Altman, D.G. Analysing Data and Undertaking Meta-Analyses. In Cochrane Handbook for Systematic Review of Interventions; Higgins, J.P.T., Ed.; Wiley-Blackwell: Hoboken, NJ, USA, 2011; pp. 243-296.

23. Buskila, D.; Abu-Shakra, M.; Neumann, L.; Odes, L.; Shneider, E.; Flusser, D.; Sukenik, S. Balneotherapy for fibromyalgia at the Dead Sea. Rheumatol. Int. 2001, 20, 105-108. [CrossRef]

24. De Andrade, S.C.; de Carvalho, R.F.; Soares, A.S.; de Abreu Freitas, R.P.; de Medeiros Guerra, L.M.; Vilar, M.J. Thalassotherapy for fibromyalgia: A randomized controlled trial comparing aquatic exercises in sea water and water pool. Rheumatol. Int. 2008, 29, 147-152. [CrossRef] [PubMed]

25. Evcik, D.; Kızılay, B.; Gökçen, E. The effects of balneotherapy on fibromyalgia patients. Rheumatol. Int. 2002, 22, 56-59. [CrossRef] [PubMed] 
26. Dönmez, A.; Karagülle, M.Z.; Tercan, N.; Dinler, M.; Issever, H.; Turan, M.; Karagülle, M. SPA therapy in fibromyalgia: A randomised controlled clinic study. Rheumatol. Int. 2005, 26, 168-172. [CrossRef] [PubMed]

27. Ardıç, F.; Özgen, M.; Aybek, H.; Rota, S.; Çubukçu, D.; Gökgöz, A. Effects of balneotherapy on serum IL-1, PGE2 and LTB4 levels in fibromyalgia patients. Rheumatol. Int. 2006, 27, 441-446. [CrossRef] [PubMed]

28. Kocyigit, B.F.; Gur, A.; Altindag, O.; Akyol, A.; Gursoy, S. Comparison of education and balneotherapy efficacy in patients with fibromyalgia syndrome: A randomized, controlled clinical study. Agri 2016, 28, 72-78. [CrossRef]

29. Bağdatlı, A.O.; Donmez, A.; Eröksüz, R.; Bahadır, G.; Turan, M.; Erdoğan, N. Does addition of 'mud-pack and hot pool treatment' to patient education make a difference in fibromyalgia patients? A randomized controlled single blind study. Int. J. Biometeorol. 2015, 59, 1905-1911. [CrossRef] [PubMed]

30. Özkurt, S.; Dönmez, A.; Karagülle, M.Z.; Uzunoglu, E.; Turan, M.; Erdoğan, N. Balneotherapy in fibromyalgia: A single blind randomized controlled clinical study. Rheumatol. Int. 2011, 32, 1949-1954. [CrossRef]

31. Fioravanti, A.; Perpignano, G.; Tirri, G.; Cardinale, G.; Gianniti, C.; Lanza, C.E.; Loi, A.; Tirri, E.; Sfriso, P.; Cozzi, F. Effects of mud-bath treatment on fibromyalgia patients: A randomized clinical trial. Rheumatol. Int. 2007, 27, 1157-1161. [CrossRef]

32. Fioravanti, A.; Manica, P.; Bortolotti, R.; Cevenini, G.; Tenti, S.; Paolazzi, G. Is balneotherapy effective for fibromyalgia? Results from a 6-month double-blind randomized clinical trial. Clin. Rheumatol. 2018, 37, 2203-2212. [CrossRef] [PubMed]

33. Pérez-Fernández, M.R.; Calvo-Ayuso, N.; Martínez-Reglero, C.; Salgado-Barreira, Á.; López-Álvarez, J.L.M. Efficacy of baths with mineral-medicinal water in patients with fibromyalgia: A randomized clinical trial. Int. J. Biometeorol. 2019, 63, 1161-1170. [CrossRef] [PubMed]

34. Zijlstra, T.R.; Van De Laar, M.A.F.J.; Moens, H.J.B.; Taal, E.; Zakraoui, L.; Rasker, J.J. Spa treatment for primary fibromyalgia syndrome: A combination of thalassotherapy, exercise and patient education improves symptoms and quality of life. Rheumatology 2005, 44, 539-546. [CrossRef] [PubMed]

35. Häuser, W.; Ablin, J.; Perrot, S.; Fitzcharles, M.-A. Management of fibromyalgia: Practical guides from recent evidence-based guidelines. Pol. Arch. Intern. Med. 2017, 127, 47-56. [CrossRef]

36. Burckhardt, C.S.; Clark, S.R.; Bennett, R.M. The fibromyalgia impact questionnaire: Development and validation. J. Rheumatol. 1991, 18, 728-733.

37. Altan, L.; Bingöl, U.; Aykaç, M.; Koç, Z.; Yurtkuran, M. Investigation of the effects of pool-based exercise on fibromyalgia syndrome. Rheumatol. Int. 2003, 24, 272-277. [CrossRef]

38. Falagas, M.E.; Zarkadoulia, E.; Rafailidis, P.I. The therapeutic effect of balneotherapy: Evaluation of the evidence from randomised controlled trials. Int. J. Clin. Pract. 2009, 63, 1068-1084. [CrossRef]

39. Langhorst, J.; Musial, F.; Klose, P.; Hauser, W. Efficacy of hydrotherapy in fibromyalgia syndrome-A meta-analysis of randomized controlled clinical trials. Rheumatology 2009, 48, 1155-1159. [CrossRef]

40. Hedenberg-Magnusson, B.; Ernberg, M.; Alstergren, P.; Kopp, S. Effect on prostaglandin E2 and leukotriene B4 levels by local administration of glucocorticoid in human masseter muscle myalgia. Acta Odontol. Scand. 2002, 60, 29-36. [CrossRef]

41. Hauser, W.; Ablin, J.; Fitzcharles, M.A.; Littlejohn, G.; Luciano, J.V.; Usui, C.; Walitt, B. Fibromyalgia. Nat. Rev. Dis. Primers 2015, 1, 15022. [CrossRef]

42. Varrassi, G.; Pergolizzi, J.V.; Dowling, P.; Paladini, A. Ibuprofen Safety at the Golden Anniversary: Are all NSAIDs the Same? A Narrative Review. Adv. Ther. 2020, 37, 61-82. [CrossRef] [PubMed]

43. O'Hare, J.P.; Heywood, A.; Summerhayes, C.; Lunn, G.; Evans, J.M.; Walters, G.; Corrall, R.J.; Dieppe, A.P. Observations on the effect of immersion in Bath spa water. BMJ 1985, 291, 1747-1751. [CrossRef] [PubMed]

44. Fioravanti, A.; Cantarini, L.; Guidelli, G.M.; Galeazzi, M. Mechanisms of action of spa therapies in rheumatic diseases: What scientific evidence is there? Rheumatol. Int. 2011, 31, 1-8. [CrossRef]

45. Becker, B.E. The Biologic Aspects of Hydrotherapy. J. Back Musculoskelet. Rehabilit. 1994, 4, 255-264. [CrossRef] [PubMed]

46. Joung, J.-Y.; Lee, N.-H.; Son, C.-G. An Evolutionary Theory to Explain the Mechanism of Pain Control by Acupuncture Therapy. J. Altern. Complement. Med. 2018, 24, 743-744. [CrossRef]

47. Mooventhan, A.; Nivethitha, L. Scientific evidence-based effects of hydrotherapy on various systems of the body. N. Am. J. Med. Sci. 2014, 6, 199-209. [CrossRef]

48. Bender, T.; Bariska, J.; Vághy, R.; Gomez, R.; Kovács, I. Effect of Balneotherapy on the Antioxidant System-A Controlled Pilot Study. Arch. Med. Res. 2007, 38, 86-89. [CrossRef]

49. Rekatsina, M.; Paladini, A.; Piroli, A.; Zis, P.; Pergolizzi, J.V.; Varrassi, G. Pathophysiology and Therapeutic Perspectives of Oxidative Stress and Neurodegenerative Diseases: A Narrative Review. Adv. Ther. 2020, 37, 113-139. [CrossRef] [PubMed]

50. Morer, C.; Roques, C.-F.; Françon, A.; Forestier, R.; Maraver, F. The role of mineral elements and other chemical compounds used in balneology: Data from double-blind randomized clinical trials. Int. J. Biometeorol. 2017, 61, 2159-2173. [CrossRef] 